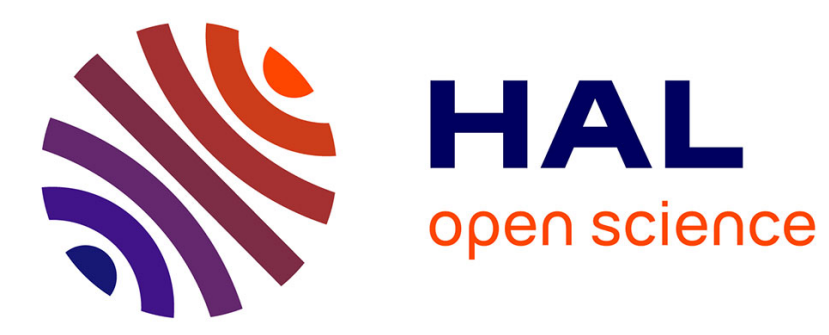

\title{
Nano- and Micromechanics of Crystalline Polymers
}

\author{
Andrzej Galeski, G Regnier
}

\section{To cite this version:}

Andrzej Galeski, G Regnier. Nano- and Micromechanics of Crystalline Polymers. Nano- and Micromechanics of Polymer Blends and Composites, Hanser Fachbuchverlag, pp.1-58, 2009, 978-3-446-41323-8. hal-02626024

\section{HAL Id: hal-02626024 \\ https://hal.science/hal-02626024}

Submitted on 26 May 2020

HAL is a multi-disciplinary open access archive for the deposit and dissemination of scientific research documents, whether they are published or not. The documents may come from teaching and research institutions in France or abroad, or from public or private research centers.
L'archive ouverte pluridisciplinaire HAL, est destinée au dépôt et à la diffusion de documents scientifiques de niveau recherche, publiés ou non, émanant des établissements d'enseignement et de recherche français ou étrangers, des laboratoires publics ou privés. 


\section{Nano- and micromechanics of crystalline polymers}

Andrzej Galeski*\#, Gilles Regnier\#

*Centre of Molecular and Macromolecular Studies, Polish Academy of Sciences, Sienkiewicza 112, 90363 Lodz, Poland

\#Arts et Métiers ParisTech, Laboratoire d'Ingénierie des Matériaux-LIM,

151 bld de l'Hopital, 75013 Paris, France

\section{Introduction}

It is currently thought that crystalline polymers consists of lamellar crystals which are separated from each other by a layer of amorphous polymer and are held together by tie molecules through the amorphous phase [e.g. 1]. The lamellae are formed from mostly folded chains. The thickness of lamellae is determined by the parameters such as interfacial energies, glass transition temperature and melting temperature, undercooling, segmental diffusivity, etc. The thickness reported lies usually in a narrow range between 3 and $20 \mathrm{~nm}$ as obtained from observations in various types of microscopes or calculated from the degree of crystallinity and long period. It has been recognized that chain folding is not so regular as it was thought and molecular packing in lamellae is subject to considerable and irregularly distributed disorder depending on undercooling- regimes of crystallization. It has been demonstrated in various ways that the planar growth front will always break up into fibrous or cellular growth. Also crystallization of polymers leads to interface instability. More sophisticated treatment of the instabilities involve perturbation analyses of planar interfaces, correlating diffusion, temperature gradients along the interface, and interfacial energy with the size of the growing crystals. All approaches show that all planar interfaces will develop and will yield fibrous or ribbonlike crystals [2 - 6]. When the crystals get larger they become stable and originate the growth of spherulites. The arrangement of lamellae within the spherulite can be best described based on the example of polyamide 6 spherulites [7]. The lamellae in bulk polyamide 6 are very long, flat and narrow. One can easily distinguish bundles of lamellae having similar orientation of flat faces and forming separate domains. Three to four lamellae form a small domain having similar orientation. As the spherulite is growing new lamellae are originated to fill the volume. New lamellae are added either to existing domain or originate new domain. The amorphous material is incorporated evenly between lamellae in the amount corresponding to overall crystallinity. Misfit between domains arising from their different shapes are filled with amorphous material. Occasionally the lamellae may terminate; usually, however, once nucleated they continue to grow until impingement with neighboring 
spherulites. New lamellae are formed mostly by non-crystallographic branching, by "giant" crystallographic dislocations and/or by crystallographic branching like in the case of isotactic polypropylene. In a number of polymers the stacks of lamellae are twisted to form banded spherulites.

\section{Tensile deformation of crystalline polymers.}

The mechanisms of tensile deformation of semicrystalline polymers were subject of intensive studies in the past [8-20]. It is believed that initially tensile deformation includes straining of molecular chains in the interlamellar amorphous phase which is accompanied by lamellae separation, rotation of lamellar stacks and interlamellar shear. At the yield an intensive chains slip in crystals is observed leading shortly to fragmentation but not always to disintegration of lamellae. Fragmentation of lamellae proceeds with deformation and the formation of fibrils is observed for large strains [21-24].

In many papers concerning tensile deformation it was noticed that shortly after yielding some cavities are formed in a polymer [11, 12, 25-27]. The cavitation is not always observed in tensile tests, i.e. the process depends on the material and testing conditions and is never observed in compression of the same material [9]. It was shown in the past that a lamellae fragmentation and cavitation during tensile drawing are often detected simultaneously [21, 28, 29], however, it was never proved whether the lamellae fragmentation causes cavitation or vice versa. Since the cavitation appears around yield (at $4-12 \%$ of strain) while lamellae fragmentation in tensile drawing occurs at the strain of 30-40\% [30] these two phenomena cannot be related directly. It seems more probable that the lamellae fragmentation is triggered by cavitaties when they reach a certain critical size. The lamellae fragmentation causes a release of 3D-stress, hence, limiting the possibility of further cavitation after fragmentation of lamellae.

\section{Cavitation in tensile deformation}

In the past the cavitation process was treated in literature as a marginal effect, however, recent studies showed that it may play an important role in the mechanism of plastic deformation $[9,10,17]$.

Usually the cavitation is detected by a small angle X-ray scattering (SAXS), seen as a rapid increase of scattering intensity. Macroscopically the intensive voiding is also observed as whitening of a deformed material. 
Liu et.al. [13] noticed that the whitening of PP during tensile deformation was present in specimens drawn more quickly and at lower temperatures, i.e. when the yield stress was higher. The stress-whitening in tensile samples of polyolefins did not occur when the test is conducted under high pressure [14, 15]. Yamaguchi and Nitta [16] investigated the intensity of light transmittance while deforming polypropylene. They concluded that the stresswhitening is observed just beyond the yield point, however, a decrease of transmittance begun at a strain of 0.07 , i.e. before yielding at strain of 0.15 .

Zhang et al. [12] analysed mechanisms of deformation for polypropylene differently irradiated, tested at selected temperatures. Authors concentrated mostly on polymer microdeformation but evidenced the voids presence in most of samples. They observed that non irradiated polymer cavitates shortly after the yield, when the temperature was below $60^{\circ} \mathrm{C}$. The SAXS scattering patterns indicate that the cavities change their shape during drawing from initially a peanut shell like to lemon like. Zhang et al. [12] proposed two explanations of shape change: a combination of scattering from primary cavities and a scattering from the voids formed during fibrillation, or primary cavities are elongated to form the voids usually associated with fibrillation.

Some authors observed in drawn polypropylene a presence of craze-like features [31-35]. Crazing was identified long time ago in many glassy polymers, such as polystyrene. Crazes have a shape of planar cracks perpendicular to local strain direction [36-38]. The edges of a crack are bridged by highly oriented polymer fibrils, usually called tufts. They are parallel to the strain direction and are able to carry the load. The thickness of crazes is in the nanometer range, similarly as the thickness of fibrils. Crazes and fibrils can be detected by small angle X-ray scattering due to nanometer range of their sizes. The characteristic SAXS pattern consists of two components originating from voids and from fibrils, in a form of two perpendicular streaks [36]. One of those streaks, very intense originating from planar crazes, is normal to craze planes while the second, usually less intense, is perpendicular to craze fibrils [36-37].

Crazing is a massive phenomena involved in plastic deformation of glassy polymers. The features of craze-like structures observed in tensile drawing of PP at temperatures higher than the glass transition temperature are significantly different. First, their thickness is larger, in the range of 1-5 $\mu \mathrm{m}$. Fibrils inside crazes, 200-650 nm thick [31], are elongated in the deformation direction, however, a significant fraction of thinner fibrils $(20-50 \mathrm{~nm})$ deviate from the principal strain direction [33]. Jang et.al. [33] showed that at $23{ }^{\circ} \mathrm{C}$ crazing is observed for strain rates higher than $0.11 \mathrm{~s}^{-2}$. Below this limit the deformation mechanism is 
the shear yielding [33]. Henning et.al [34] by microscopic observations identified positions of intense crazing in deformed spherulitic structure. The conclusion was that craze-like features are visible for $\alpha$ - polypropylene in polar regions of spherulites. Some large scale, transspherulitic crazes were observed by Narisawa et.al. [35] passing through equatorial parts of spherulites.

Friedrich [31] and later Kausch [32] reviewed crazing processes in semicrystalline polymers. In their opinion it involves several stages: the initial stage is generally a macroscopically homogeneous deformation involving lamellar tilt and some breakup. At this stage the strain is accommodated almost entirely by the interlamellar amorphous regions [31]. Disruption of lamellae into 10-30 nm size blocks, voiding (with a length of voids from 10 to 50 and their thickness from 2 to $6 \mathrm{~nm}$ ) and formation of fibrils between voids from partly extended tie molecules and crystal blocks characterize the intermediate stage. The third stage, at higher draw ratios, leads to a perfect stretching of the fibrils. The fibrils, spanning the edges of a craze and possessing the strength of about 1 to 2 orders higher than the yield stress, are able to stabilize the microvoid volume. A lateral coalescence of these voids finally provides a local deformation zone in the shape of a craze. Friedrich limited this description to a low temperature crazing. The Kausch description [32] differs from Friedrich envision in that that in the first stage the interlamellar separation of lamellae oriented perpendicularly to deformation direction is followed by cavitation and/or crystal plastic deformation and not lamellar fragmentation. Lamellar fragmentation occurs at the intermediate stage.

In an earlier paper by Galeski, Argon and Cohen [39] the following scenario of crystalline polymer deformation under the imposed uniaxial tension was outlined: the packets of lamellae in the $45^{\circ}$ fans of spherulites experience resolved shear stress that promote chain slip in the lamellae and shear in interlamellar amorphous regions (see Figure 1).
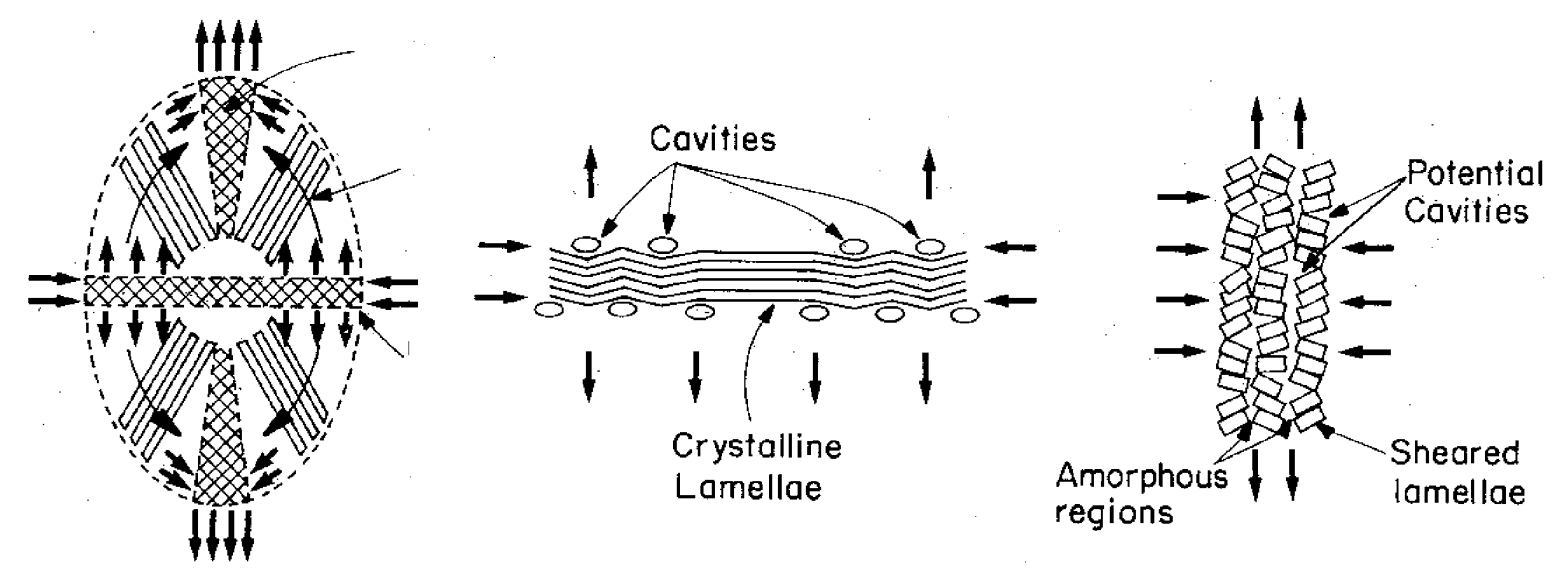
Figure 1. Tensile deformation of a spherulite. Lamellae kinking and formation of cavities in equatorial zones of a spherulite are formed. Lamellae fragmentation occurs in polar fans of spherulites, potential cavities may be also expected . Redrawn after [39].

Such deformation is accompanied by lattice rotation which generates tensile stresses across the faces in equatorial plates and compressive stresses across the faces in polar fans of spherulites. In addition to these, the overall elongation of spherulites evokes radial pressure on the equatorial planes and additional tensile stresses in the radial direction on the polar fans. Since these accentuated stresses in equatorial lamellae packets have no important shear components either on the interlamellar layers or on the planes of lamellae that promote chain slip, other more damaging types of local plastic deformation are enforced. As is well known [40] compression of such composite stacks lamellae and amorphous layers along stiff lamellae gives rise to unstable kinking of the lamellae. This produces periodic undulations in the lamellae ribbons. The accentuated tensile stresses acting across the equatorial disks of a spherulite expand amorphous material within lamellae kinks into pores. A similar and complementary processes are expected to occur in a polar fans of spherulites, however, no action is expected from the amorphous phase, but instead, from the lamellae ribbons. In this case the chain fold planes in the lamellae form an unstable stacks in tension. Any inhomogeneous lamellae kinks would be filled with amorphous material under transverse pressure, producing no voids. Thus, the end result should be the array of aligned cavities in the equatorial disks of spherulites.

The above outlined picture of voiding in crystalline polymers above $\mathrm{T}_{\mathrm{g}}$ differs significantly from craze formation in glassy amorphous polymers:

1. Formation of aligned voids is triggered by compressive kinking instability along lamellae in equatorial disks of spherulites and not by meniscus instability as in the case of crazes in glassy amorphous polymers. Distances between voids are characteristic for kinking that is dependent on several factors, the most important being the lamellae thickness [41]. The wavelength of kinks in crystalline polymers is usually of order of 200-500 nanometers. The voids are narrow and long as the width of kinked fragments of lamellae.

2. Growth of craze-like entities in equatorial disks of spherulites occurs via plastic deformation of the amorphous material between laterally aligned voids that is transformed further into fibrils. The thickness and length of these fibrils is related to the amount of the amorphous material between voids and that is connected in the first 
approximation with the lamellae thickness. These fibrils may mimic tufts in crazes of amorphous glassy polymers.

3. Voids in other parts of a spherulite arise at later stages of deformation and the mechanisms of their formation is different. Since there are no dilatational stresses at $45^{\circ}$ fans of spherulites the interlamellar slip and chain slips in lamellae prevails and no voiding is usually observed.

4. A disruption of lamellae in polar fans of spherulites is required, pores are formed at larger overall strain when the amorphous material becomes unable to fill significantly increased gaps between lamellae fragments. At even further strain the neighbouring voids coalesce in planes perpendicular to drawing direction while the amorphous material between these voids is transformed into fibrils. The thickness of those fibrils is related to the thickness of the amorphous layers and depends on the strain at which the voids coalesce and fibrils are formed. These craze-like entities resemble rather thick cracks because fibrils usually break at such high local strain.

The above concept of formation of craze-like objects in crystalline polymers explains the existence of bimodal distribution of fibril thickness and is also supported by recently published AFM studies for initiation of voids in polybutene spherulites and their transformation into craze-like entities with increasing deformation [42]. The craze-like features in polypropylene are seen by electron microscopy, however, in contrast to crazing in glassy amorphous polymers, the fibrils in PP are usually not detectable in SAXS experiments probably due to a small number of fibrils and their large thickness.

Some SEM observations (e.g. Jang, B.Z et.al. [33]) show fibrils in drawn PP only after etching. However, it is not clear whether these are craze tufts or remnants of initial crosshatched PP lamellae revealed by etching. The last case seems more reasonable because there are two populations of fibrils, the second one is nearly perpendicular to the first one just as in cross-hatched morphology.

In some other papers (e.g. [19]) the term "crazing" is misused for the description of microcracks.

Formation and growth of cavities are the main reasons of volume change during tensile drawing. The concept of volume strain, introduced by Bucknall [43], was recently applied for the studies of semicrystalline polymers [44-48]. The volume strain of cavitating polymers may significantly increase with increasing draw ratio, and reach 0.4 for PET [45] and more than 0.15 for PVDF [46]. Recently, Billon et.al. [49] observed a rapid increase of volume 
strain for PP, resulting from nucleation of voids in the core of injection molded polypropylene samples.

It was shown that for most of crystalline polymers including polypropylene and other polyolefins the tensile drawing proceeds at a much lower stress than kinematically similar channel die compression $[10,17]$. Lower stress in tension was always associated with cavitation of the material. Usually a cavitating polymer is characterized by larger and more perfect lamellar crystals and cavities are formed in the amorphous phase before plastic yielding of crystals. If the lamellar crystals are thin and defected then the critical shear stress for crystal plastic deformation is resolved at a stress lower than the stress needed for cavitation. Then voiding is not activated. An example of such behavior is low density polyethylene [10].

\section{Tensile deformation of polyethylene and polypropylene.}

Recently the cavitation during tensile deformation in high density polyethylene was studied in details [50]. The samples were prepared by both injection and compression moldings in order to differentiate their internal structure and perfection of lamellae. It was shown that cavitation in HDPE depends on lamellae thickness and their arrangement. For example, when the crystals were oriented perpendicularly to drawing direction, as in the skin of injection molded specimen, the cavities were formed readily as early as at $1 \%$ of elongation (see Figure 2). 

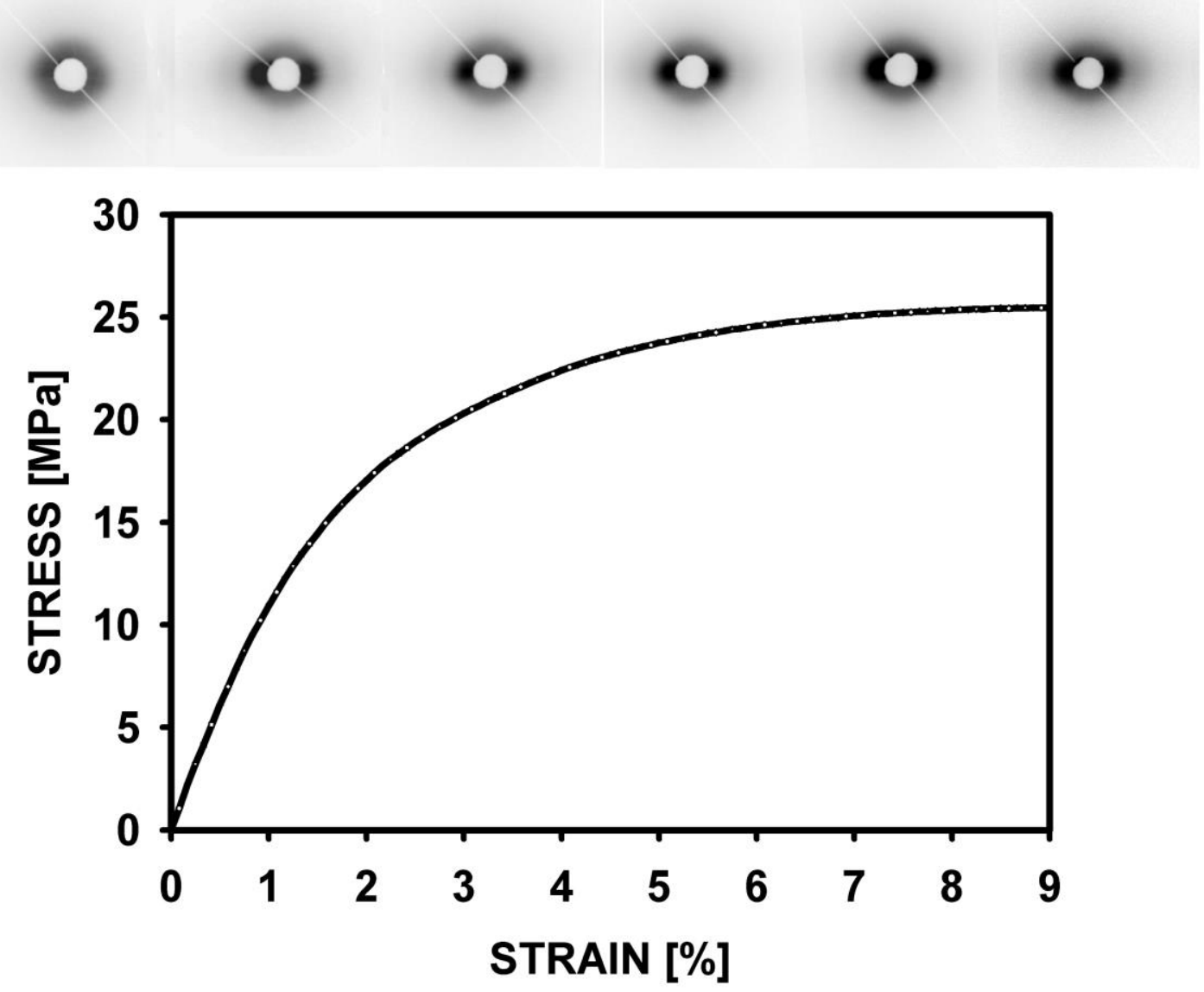

Figure 2. The evolution of SAXS scattering pattern with the increase of stress applied to the HDPE-I. Deformation direction was horizontal. Redrawn after [50].

Cavitation in the core of the same injection molded HDPE sample was observed at yield (above $10 \%$ of elongation). The shape of cavities changes with increasing deformation similarly as it was described by Zhang et.al. [12]. It was also shown that the same polymer formed by rapid cooling of melt is able to deform without cavitation during tensile drawing. The stress-strain curves for compression molded HDPE cooled in air, in water and in iced water together with respective volume strain measurements are presented in Figures $3 \mathrm{a}$ and $3 \mathrm{~b}$. 

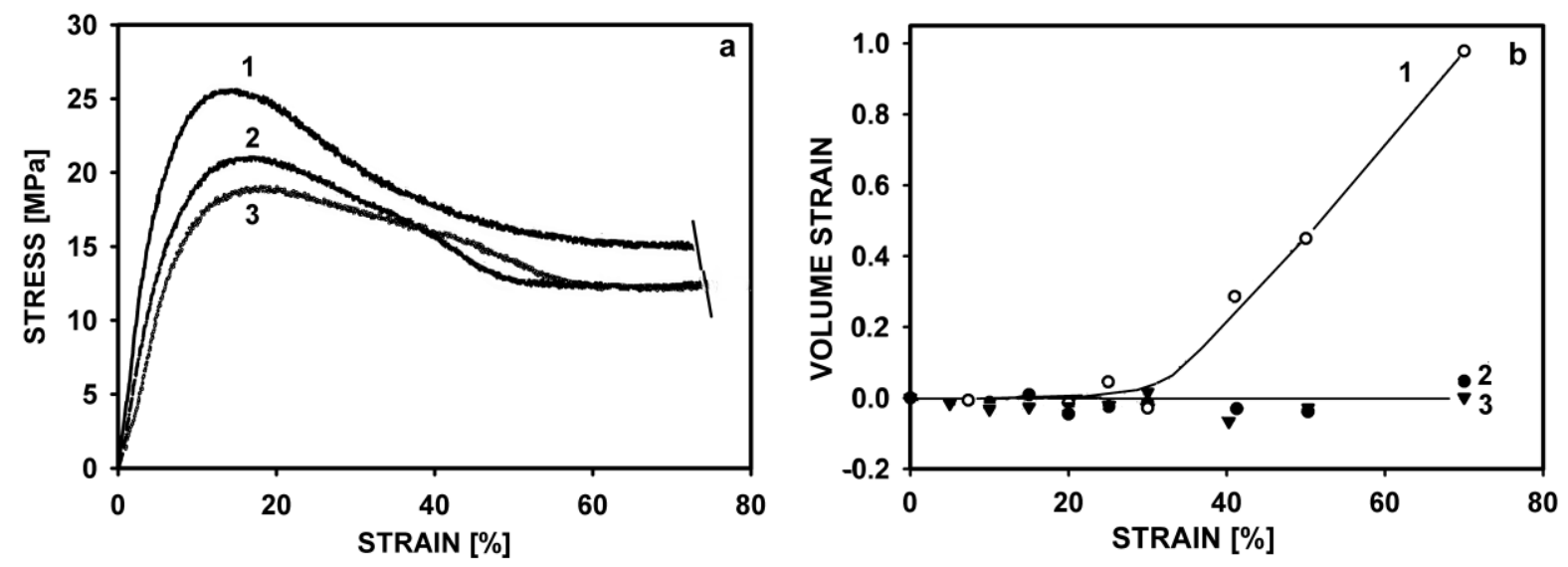

Fig 3. a) Stress-strain curves for compression molded HDPE samples, deformed to $75 \%$ of engineering strain. Different cooling procedure was applied during preparation: 1- sample cooled in the air, 2- sample cooled in the water, 3- sample cooled in the water with ice. Redrawn after [50].

Polypropylene is a cavitating polymer similar to polyethylene, but the process of cavitation proceed differently. The role of molecular weight, crystal thickness and testing rate on the formation of cavities and their growth during tensile deformation were the subjects of detailed studies [51]. The injected samples of PPH and PPN deforms plastically with necking and significant increase of the volume is observed only after the yield. The increase of volume is accompanied by intensive whitening of samples. The reason of whitening and volume increase is cavitation initiated in the amorphous phase, which was confirmed by SAXS studies. The volume strain is larger for lower molecular weight PP polymer, in which the amorphous phase is less entangled. Apparently a lower number of entanglements supports easier and more numerous cavitation. The cavities are formed during tensile drawing in the center of injected PP samples shortly before yielding. At small local strains, after the yield, a partial healing of small cavities is possible. The zone of cavitation propagates to the rest of sample with increasing deformation. In the skin of injected samples voids are not detected for lower local strains than 2.6 in contrast to the core injection molded bars of PP of lower and also higher molecular weight. That is because in the skin the crystalline structure is less developed which leads to crystallographic non-cavitational mechanism of deformation. Cavitation process occurs when the crystals are enough perfect and strong, i.e. when the stress for plastic deformation of crystals is higher than the stress needed for cavitation of the amorphous phase. At low local strain the cavities are elongated perpendicularly to deformation direction. Typical radius of gyration for voids, as obtained from SAXS analysis, in PP of higher molecular weight is $18 \mathrm{~nm}$ and $12 \mathrm{~nm}$ for voids in PP of lower molecular 
weight. If we assume ellipsoidal shape of voids and that their thickness is equal to the amorphous layer thickness (approximately $7 \mathrm{~nm}$ ) then the lateral size of cavities is around 80 $\mathrm{nm}$ in PP of higher molecular weight and around $50 \mathrm{~nm}$ in PP of lower molecular weight. The cavities grow with increasing strain and also some new voids are formed.

The volume strain does not increase continuously with deformation. There is a plateau in volume strain for applied local strains between 1.0 and 4.0, where the change of internal crystalline structure - orientation and fragmentation of lamellae- leads to a change of voids shape from elongated perpendicular to elongated in the deformation direction. Reorientation process sets in for local strains of $0.8-1.0$

The structure of compression molded samples is more uniform than the injection molded bars, so the cavitation occurs across the gauge of the sample. The presence of cavitation in those samples depends on the strain rate. If the deformation is slow then the crystalline elements are able to deform plastically before reaching the cavitation threshold of amorphous phase. When the strain rate is higher the yield stress, related to crystal strength, is higher (38-38.5 MPa) and cavitation occurs first.

The lack of fibrils bridging the walls of cavities results from the mechanism of their formation within spherulitic structure in crystalline polymers above their $\mathrm{T}_{\mathrm{g}}$ as explained in above [39]. Drawing below $T_{g}$ may produce craze-like features because inhomogeneous lamellae kinks would not be easily filled with glassy amorphous material under transverse pressure, producing voids and drawing crazy tufts in polar fans of spherulites.

The main reasons of cavitation are the misfit between mechanical compliances of heterogeneous element and the surrounding and the resulting excessive negative pressure. Cavitation appears to be another mechanism of tough response of the material [e.g 52, 55]. Although the cavitation dissipates not very large amount of energy, it enables the surrounding material to undergo further intensive crazing or shear. The possibility of cavitation under external force strongly depends on the character of applied deformation and is larger under tension than under compression. Cavitation in macro scale is visible as whitening. The voiding was observed in many drawn polymeric materials such as polypropylene [54-57] and high density polyethylene [58], but not in some others like ethylene- $\alpha$-olefin copolymer $[59,60]$. It was established in the past that a low crystallinity level and a fine spherulitic structure are advantageous for development of crazes in semicrystalline polymers [61]. Unlike in amorphous polymers crazing in semicrystalline polymers is not restricted to temperatures below $\mathrm{T}_{\mathrm{g}}$ but occurs also above the glass transition of the amorphous phase [62]. Below $\mathrm{T}_{\mathrm{g}}$ long crazes propagate perpendicularly to load direction ignoring internal polymer structure 
while at a temperature close to $T_{g}$ crazes are confined to several spherulites $[31,62,63]$. Radicals formed due to macromolecules chain scission were observed in oriented polymers during tensile deformation $[64,65]$.

The internal cavitation observed in tension experiments has been referred to as "micro necking" by Peterlin [66]. It was supposed that "micro necking" removes kinematical constraints between lamellae and allow them to untangle. However, from this picture as imagined by Peterlin, it follows that the drawing of crystalline polymers inherently involves cavitation as an essential feature. While the above picture is reasonable in tensile deformation, it is not correct for modes of deformation not producing cavitation, in which positive normal stress component prevents for the formation of cavities. "Micro necking" is inessential for the development of nearly perfect single crystal textures, as it was shown for several semi crystalline polymers that result from the deformation modes involving positive pressure component [67-72]. One of the possibilities of cavity free deformation is plane strain compression in a channel die [73]. Plane strain compression in a channel die is kinematically very similar to drawing: the sample is extended and its cross-section decreases accordingly. However, no neck is formed and the possibility of voids formation is limited due to a compressive component of stress. It is reasonable to expect that similar elementary plastic deformation mechanisms are initiated under similar true stress in tension and in plane strain compression. It means that any differences in true stress- true strain dependences of drawn and plane strained materials should be attributed to the formation and development of cavities. The number of observations of polymer deformation behavior under positive pressure at the level preventing for cavitation which can be found in literature [e.g. 14] is limited and no comparison with tensile drawing was made. It is known that the stress in heterogeneous systems is not homogeneously distributed. At sites with a misfit of mechanical properties the stress concentrates, its value may increase locally many folds and trigger cavitation. In the case of crystalline polymers such sites are between differently oriented packets of lamellae and the stress concentration factor can reach 3 or more.

In the paper by Galeski, Argon and Cohen [39] the imprints of cavitation were revealed by osmium tetroxide staining in drawn polyamide 6 . The cavitation occurred in the amorphous layers confined by the crystalline lamellae. In fact the cavities left the traces of damaged material having the size of order of the interlamellar distance, i.e. 4-5 nm. Since cavitational voids are having sizes on the nano-scale level, there is a problem of their stability. On each nanopore the surface tension is exerted from the very beginning of their formation, 
which tends to close a pore. In order to preserve a pore an action of a negative pressure is required at the level which is reciprocally proportional to the radius of a pore:

$\mathrm{p}=-2 \tau_{\mathrm{s}} / \mathrm{r}$

where $\tau_{\mathrm{s}}$ is the surface tension and $\mathrm{r}$ is the size of a pore. It follows then that the smallest pores are healed readily while larger pores can be preserved only if the negative pressure is maintained at a sufficiently high level. The lack of negative pressure or its inadequate level will lead to a spontaneous healing of cavitational pores; otherwise they can grow in the course of drawing.

In the papers by Muratoglu et al. $[74,75]$ it was shown that when cavitational pores arise they form initially a spherical voids which later grow and become elongated. It is then logical to suppose that at the moment of cavity formation the stress concentrates around a spherical inhomogeneity and, as it was calculated earlier by Goodier [76] for spherical inclusions, is increased by a factor of 2 . Also the negative pressure generated is increased accordingly.

Table I. Surface tensions [77-79] and negative pressures involved in cavitation during drawing.

\begin{tabular}{|l|l|l|l|l|l|}
\hline Polymer & $\begin{array}{l}\text { Surface } \\
\text { tension } \\
{\left[\mathrm{mJ} / \mathrm{m}^{2}\right]}\end{array}$ & $\begin{array}{l}\text { Amorphous } \\
\text { layer thickness } \\
{[\mathrm{nm}]}\end{array}$ & $\begin{array}{l}\text { Negative } \\
\text { pressure needed } \\
\text { to maintain } \\
\text { cavity open } \\
{[\mathrm{MPa}]}\end{array}$ & $\begin{array}{l}\text { Tensile yield } \\
\text { stress [MPa }]\end{array}$ & $\begin{array}{l}\text { Negative pressure } \\
\text { generated at yield } \\
\text { around newly } \\
\text { formed cavities } \\
{[\mathrm{MPa}]}\end{array}$ \\
\hline POM & 44.6 & 4.69 & -35.8 & 63 & -42.0 \\
\hline PA6 & 38.4 & 5.59 & -33.6 & 42 & -28.0 \\
\hline PP & 29.4 & 8.16 & -13.7 & 31 & -20.7 \\
\hline HDPE & 35.7 & 9.45 & -15.1 & 23 & -15.3 \\
\hline LDPEs & 35.3 & 7.50 & -17.6 & 7 & -4.7 \\
\hline
\end{tabular}

*calculated on the basis of SAXS long period and DSC degree of crystallinity.

In Table I the data on surface tension, thickness of the amorphous layers, negative pressure needed to maintain the cavity open (according to equation (1) assuming the radius of newly formed cavity equal to half of the amorphous layer thickness) and the yield stress in drawing are presented. In the last column of the Table I the negative pressure generated at yield around newly formed cavities is listed. These values of pressure result from the stress at yield ( - 
$\sigma_{\text {yield }} / 3$ ) increased by a factor of 2 arising from the stress concentration as predicted by Goodier [76].

It is clearly seen that the negative pressure at concentration sites, which is the negative pressure at yield $\left(-\sigma_{\text {yield }} / 3\right)$ multiplied by stress concentration factor 3 , can cause cavitation in the case of PA 6, POM, PP and HDPE because it is higher than the negative pressure for cavitation in polymer melts (HDPE from -3.5 to $-10 \mathrm{MPa}$ [80], PP from -13 to -19 MPa [8183], POM from -10 to -18MPa [81-83] but not adequate for cavitation of LDPEs.

In POM, PP and HDPE the yield stress generates sufficient negative pressure around cavities to maintain them and to boost their further growth. In fact the cavitation in those polymers during drawing is evidenced by density decrease and strong small angle x-ray scattering.However, it is also evident that the negative pressure generated around newly formed cavities is not sufficient to keep them open in the case of PA 6; the cavities in PA 6 are unstable and will heal quickly. Really, the transmission electron microscopy (TEM) examination [39] of the same material does not expose any empty voids but only traces of chemically changed material between crystalline lamellae. It must be mentioned here that the growth of cavities proceeds in fact due to the deformation of the surrounding matter, therefore, it is due to the action of dilatational stresses and not due to negative pressure.

\section{Deformation micromechanisms in crystalline polymers..}

There are three, currently recognized, principal modes of deformation of the amorphous material in semicrystalline polymers: interlamellar slip, interlamellar separation and lamellae stack rotation $[84,85]$. Interlamellar slip involves shear of the lamellae parallel to each other with the amorphous phase undergoing shear. It is a relatively easy mechanism of deformation for the material above $\mathrm{T}_{\mathrm{g}}$. The elastic part of the deformation can be almost entirely attributed to the reversible interlamellar slip.

Interlamellar separation is induced by a component of tension or compression perpendicular to the lamellar surface. This type of deformation is difficult since a change in the lamellae separation should be accompanied by a transverse contraction and the deformation must involve a change in volume. Hard elastic fibers are found to deform in such way. When the lamellae are arranged in the form of stacks embedded in the amorphous matrix then the stacks are free to rotate under the stress. Any other deformation of the amorphous phase requires a change in the crystalline lamellae; the amorphous material is then carried along with the deforming crystalline material. 
Many authors identified two distinct yield points in PE deformed in the tensile mode which are not seen in other deformation modes not producing cavitation. Gaucher-Miri and Seguela [86] tried to clear up the mechanism of this two processes on the micro-structural level, as a function of the temperature and strain rate.

The major contribution to toughness comes from plastic deformation of the material which is manifested by the ductile behaviour. Plastic deformation itself is a composed phenomena: it concerns the crystalline as well as amorphous phases. The ductility is expressed by lowering of the stress-strain curve at a certain stress called the yield stress. Yield can be caused either by multiple crazes or by shear yielding. In the first case the crazes have to be initiated in a relatively large volume of the material in order to contribute significantly to the overall deformation. Shear yielding is the plastic flow without crazing. Crazing is an unique phenomena occurring in polymers below the glass transition temperature. Crazes themselves are highly localized zones of plastic dilatational deformation [e.g.87-90]. Edges of crazes are spanned by highly drawn elongated fibrils called tufts, usually having the length of a fraction of $1 \mu \mathrm{m}$ depending on the molecular weight of a polymer, the diameter of several nanometers and confined to a small volume of the material. The tufts can carry the load applied to the material and preserve its integrity. In brittle materials crazes are initiated at surfaces. Brittle fracture is usually caused by microcracks originating from breaking crazes initiated from the surface. Crazing occurs mostly in amorphous polymers although it has been also observed in crystalline polymers in which crazes are propagated between lamellae through spherulite centers as well as through the material between spherulites [91,92]. Localized crazes initiate, propagate and break down to give microcracks at the stress below that which is necessary to stimulate shear yielding

Shear yielding can be observed in a wide range of temperature but only if the critical shear stress for yielding is lower that the stress required to initiate and propagate crazes.

Ductile deformation requires an adequate flexibility of polymer chain segments in order to ensure plastic flow on the molecular level. It is long known that the macromolecular chain mobility is a crucial factor deciding on either brittle or ductile behavior of a polymer [93-95]. An increase in the yield stress of a polymer with a decrease of the temperature is caused by the decrease of macromolecular chain mobility, and vice versa, the yield stress can serve as a qualitative measure of macromolecular chain mobility. It was shown that the temperature and strain rate dependencies of the yield stress are described in terms of relaxation processes, similarly as in linear viscoelasticity. Also the kinetic elements taking 
part in yielding and in viscoelastic response of a polymer are similar: segments of chains, part of crystallites, fragments of amorphous phase. However, in crystalline polymers above their glass transition temperature the yield stress is determined by the yield stress required for crystal deformation and not by the amorphous phase. The behavior of crystals differs from that of the amorphous phase because the possibilities of motion of macromolecular chains within the crystals are subjected to severe constraints. Since the mobility of kinetics elements taking part in a plastic deformation is lower at a lower temperature, the energy dissipated increases and produces instability; at those places micronecks are formed because locally the temperature increases. The rate of plastic deformation increases drastically in micronecks and the material may quickly fracture. At higher temperature the mobility of kinetics elements is higher so, less energy is dissipated and the local temperature increase is lower. As the result the neck is stable and tends to occupy the whole gauge length of the sample. The material exhibits then a tough behavior.

The necessary condition for high plastic deformation is the possibility of motions of kinetic elements in a time scale as it follows from the deformation rate. The relaxation times and the activation energies are the parameters describing the kinetics of the conformation motions of macromolecules and larger elements taking part in the deformation. In crystalline polymers there are essentially three processes which are of particular importance: the first process which is connected with the presence of crystalline phase, the second corresponding to relaxations related to defects in the crystalline phase and the third which corresponds to motions of short segments in the amorphous phase related to the glass transition [94] .

Both massive crazing and shear yielding dissipate energy, however, shear yielding is often favored over crazing, especially under uniaxial stress, elevated temperature or slow deformation. Shear yielding dissipates the energy more efficiently [94]. Switching between crazing and shear yielding is not obvious as it depends also on additional factors such as shape of an article and the presence of notches or scratches. If all other factors are equal the material will deform according to a most ductile mechanism which is well explained by the Ludwig-Davidenkov-Orovan hypothesis [96-98] explained in Figure 4. 


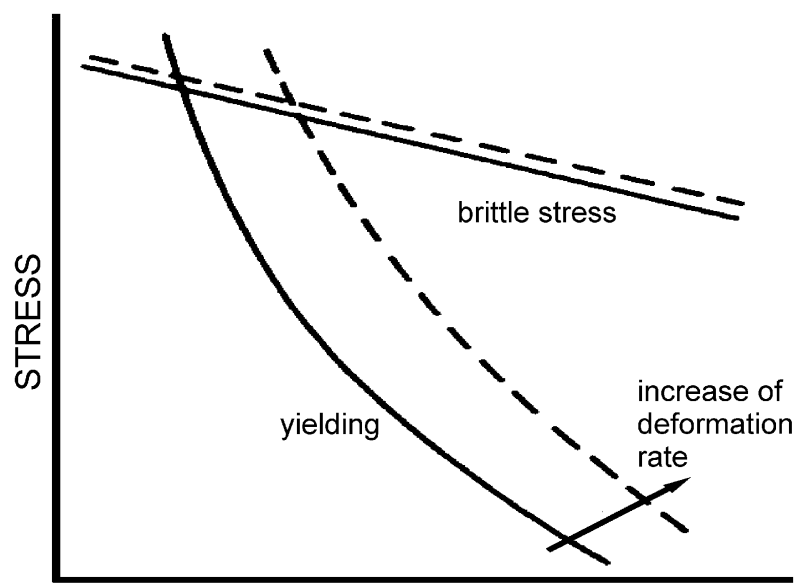

TEMPERATURE

Fig.4 Ludwig-Davidenkov-Orovan plot explaining the temperature dependence of ductility and brittleness of a material. Redrawn after Ward and Hadley [99].

The deformation of polymeric materials starts usually at scratches, notches or internal defects because these places are the zones of local stress concentration, sometimes much above the applied stress. Toughening of polymeric materials is based on the activation of such plastic deformation mechanisms which are activated at a stress lower than that required for triggering the action of surface and internal defects. Consequently, one of the important means of toughening appears to be a significant lowering of yield stress of a material [100].

\section{Molecular mechanisms at a nanometer scale.}

Taking into consideration the yield behavior of semicrystalline polymers there are two conflicting approaches concerning the crystals. The first presumes that the process of deformation is composed of a simultaneous melting and recrystallization of polymer under adiabatic conditions [101,102]. The second, developed mainly by Young [103] uses the idea derived from the classical theory of crystal plasticity. The deformation of polymer crystals is considered in terms of dislocation motion within the crystalline lamellae, similarly to slip processes observed in metals, ceramics and low molecular crystals. Crystallographic slips are not processes occurring simultaneously over the whole crystallographic plane. A great role of line and screw dislocations are played in activation and propagation of a slip. Recently the issue of melting-recrystallization vs.crystal plasticity was raised again by Seguela [104]. His conclusion is that melting-recrystallization may occur at high strain rates, during lamellae fragmentation and at high strain when collective chain unfolding takes place. However, most of published data concern the systems in which plastic deformation is accompanied by cavitation-the phenomena neglected by most of the authors. Also Seguela in his revoking 
review [104] does not refer to the relation between lamellae fragmentation and cavitation. Recent papers of Strobl [30], Lv et al. [105] and Pawlak et al. [51] present the attempts to correlate the two coexisting phenomena.

The plastic deformation of polymer crystals, like the plastic deformation of crystals of other materials, is generally expected to be crystallographic in nature and to take place without destroying the crystalline order. The only exception to this is a very large deformation, when cavitation and voiding lead to unravelling the folded chains and break down completely the crystals, new crystals may form with no specific crystallographic relationship with the original structure [106]. Polymer crystals can deform plastically by crystallographic slip, by twinning and by martensitic transformation. The slip mechanism is the most important one since it can produce larger plastic strains than the other two mechanisms. A slip system in a crystal is the combination of a slip direction and a slip plane containing that direction as shown in Figure 5.

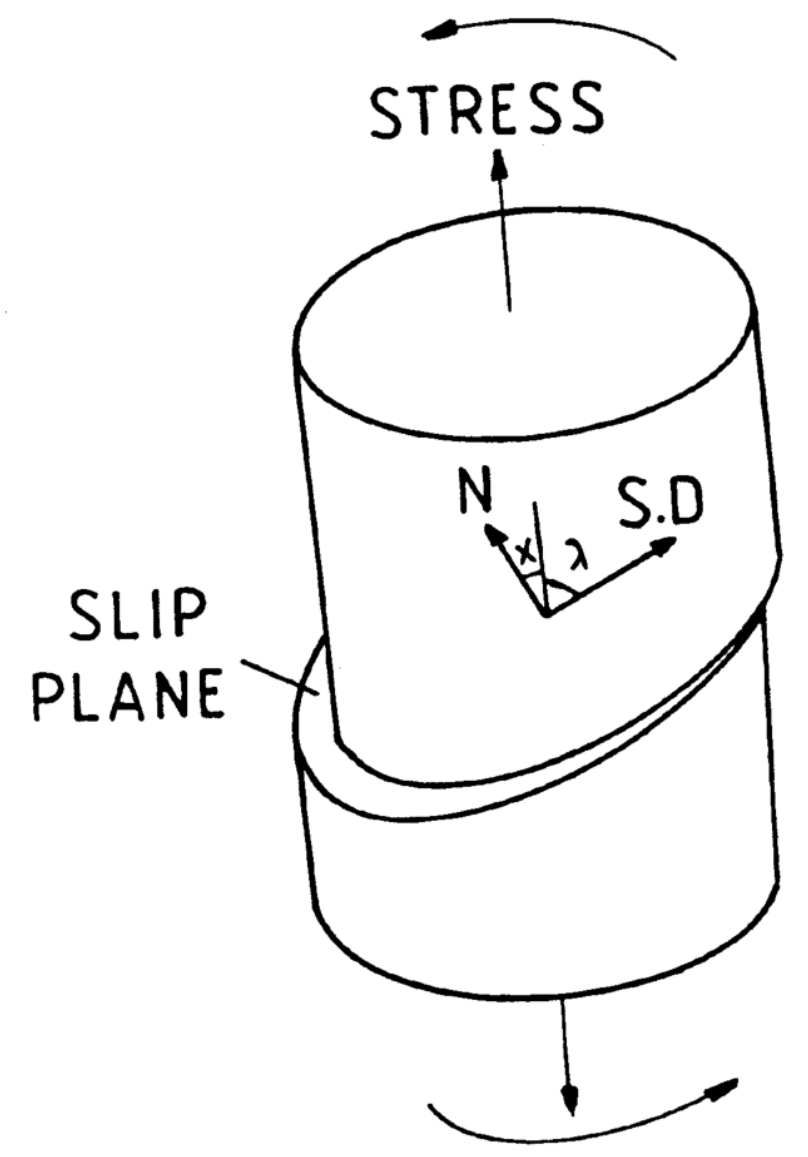

Figure 5. Definition of a slip system: slip plane and slip direction 
The notation for the slip system is $(h k l)\left[h_{1} k_{1} l_{1}\right]$ where (hkl) is the slip plane while $\left[h_{1} k_{1} l_{1}\right]$ is the slip direction. A single slip system is only capable of producing a simple shear deformation of a crystal. A general change of a shape of a crystal requires the existence of five independent slip systems [107]. Polymer crystals rarely possess this number of independent slip systems. However, under the right conditions the deformation of bulk material can occur without voiding or cracking perhaps because amorphous regions between lamellae allow for certain amount of adjustment. In polymer crystals the slip plane is restricted to planes which contain chain direction. That is because covalent bonds remain unbroken during deformation. In polymers two types of slip can occur: chain slip i.e. slip along the chains and transverse slip i.e. the slip perpendicular to the chains both slips occurring in planes containing the chains. The general rule that applies to slip deformation is that the plane of the easiest slip tends to be a close-packed plane in the structure and the slip direction is a close-packed direction. Hence, in crystalline material, it is possible to predict certain mechanical properties associated with crystallographic slip directly from the crystallographic unit cell [108]. In folded chain polymer crystals the folds at the surface of crystals may in addition impose some restraint on the choice of a slip plane; usually a slip will be able to occur only parallel to the fold plane. An implication of the geometry of the slip process is that a crystal undergoing a single slip will rotate relative to the stress axis as it is seen in Figure 6. 

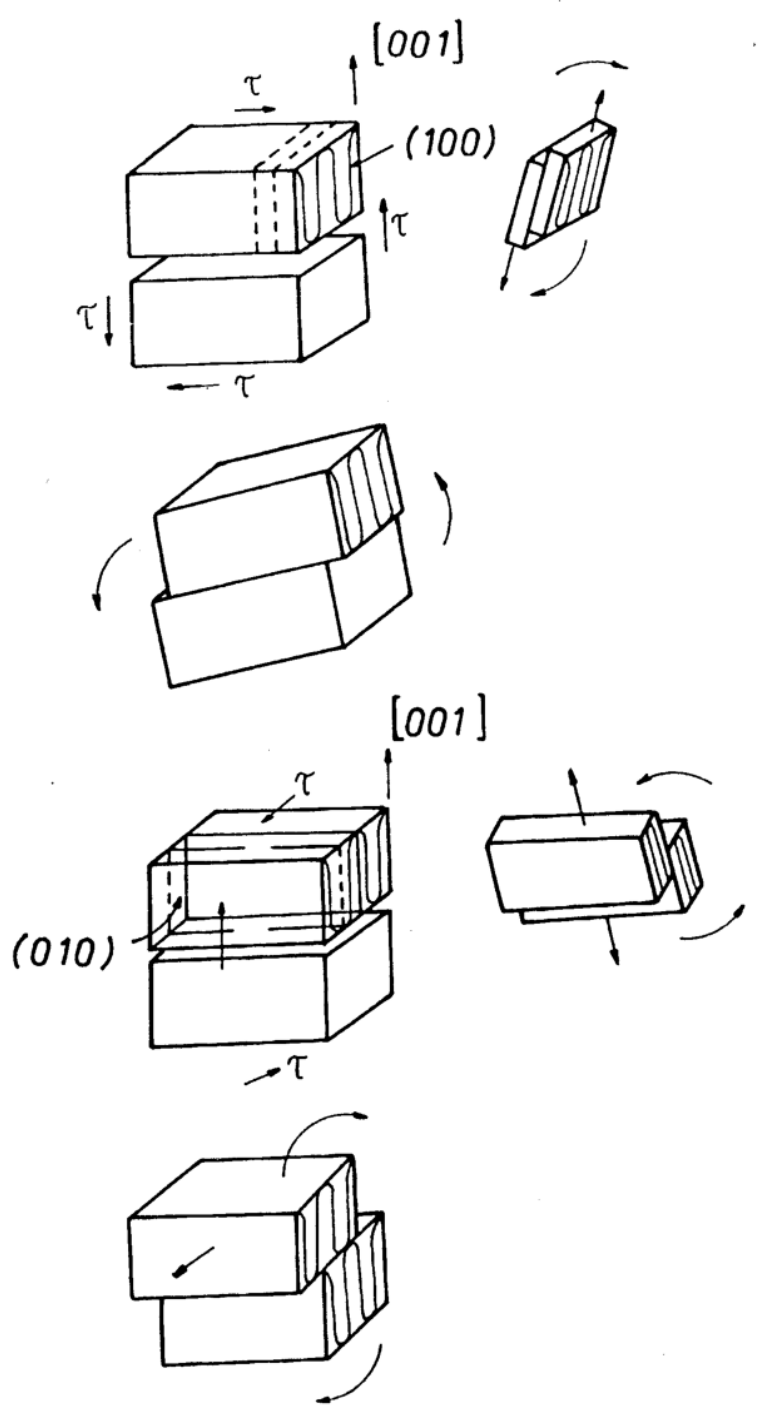

Figure 6. The rotation of crystal fragments due to slip: marked by arrows. The resolving of the shear on a plane due to simple tension or compression is also illustrated.

For a single slip the slip direction in the crystal rotates always towards the direction of maximum extension: in uniaxial tension it rotates towards tensile axis while in uniaxial compression away from the compression axis. The angle through which the crystal rotates is a simple function of the applied strain [107]. It must be mentioned that the slip takes place when the resolved shear stress on the slip plane reaches a critical value known as a critical resolved shear stress. The critical resolved shear stresses for slips are now well known only for few polymers.

They were measured using samples of rather well defined texture. First measurements performed on polyethylene sample with fiber texture subjected to annealing at high pressure for increasing the crystal thickness yielded the critical shear stress of $11.2 \mathrm{MPa}$ for 
undisclosed crystal thickness [84]. However, with fiber symmetry a combination of easiest slip systems could act simultaneously disturbing the true value of the critical shear stress. The most exact data concerning critical resolved shear stresses for possible slip systems in polyethylene were obtained by Bartczak et al. [109]. They used single crystal textured polyethylene obtained by plane strain compression. The measurements in uniaxial tension, uniaxial compression and simple shear for samples cut out at various orientations delivered the following: for polyethylene orthorhombic crystals [109] the most active slip system is (100)[001] chain slip at 7.2 MPa, the second is (100)[010] transverse slip at $12.2 \mathrm{MPa}$, and the third is (010)[001] chain slip at 15.6 MPa. The forth slip system for polyethylene orthorhombic crystals was predicted as (110)[001] with the estimated critical resolved shear stress greater than 13.0 MPa [109], however, it was never observed separately.

For polyamide $6 \alpha$ crystals the slip systems are: (001)[010] chain slip at $16.24 \mathrm{MPa}$, (100)[010] chain slip at 23.23 MPa and (001)[100] transverse slip [71]. Relatively little attention was paid to the plastic deformation of other semicrystalline polymers $[98,110]$. In particular, there are only few papers $[111,112]$ describing the investigations of the yield behaviour and plastic resistance of oriented iPP.

For the determination of critical shear stress of one of the most important deformation mechanisms of iPP crystals, namely the crystallographic slip in the (100) planes along chain direction, i.e. (100)[001] chain slip [113, 114], the biaxially oriented film was used [115]. According to the theoretical predictions [113] and experimental studies [116] the easiest slip system in iPP crystals is (010)[001] slip, while (100)[001] and (110)[001] systems have higher critical resolved shear stress. The studies of the mentioned above slip system can be made by the investigation of the yield behaviour of the specimens deformed in tension with the tensile axis oriented in those specimens at various angles to the orientation direction. One can expect that for a certain range of this angle only the (100)[001] slip system will be activated due to proper orientation of crystallites providing high shear stress on the (100) plane in [001] direction, while other deformation mechanisms will remain inactive due to much smaller resolved shear stresses in appropriate directions $[109,116]$. The analysis of the yield stress of such samples would give the value of the shear stress necessary to activate the (100)[001] slip [85, 109, 110, 117]. The critical resolved shear stress for (100)[001] slip for $\alpha$ crystals of polypropylene was determined at the level of $22.6 \mathrm{MPa}$. Similar studies for oriented iPP in tension and compression were performed by Shinozaki and Groves [111] but they used the samples of uniaxially oriented iPP with a fiber symmetry, so that the critical 
shear stress they determined, 25MPa, was an average over slips in plane oriented around the fibre axis.

Crystal orientation by channel die compression of PET was studied by Bellare et al [117]. They have found that the macromolecular chains orientation in PET along the flow direction and the texture development are the results of possible crystallographic slips having the following glide planes and directions: (100)[001] chain slip and (100)[010] transverse slip and (010)[001] chain slip. Probable sequence of activities of these slips is the following: the (100)[001] chain slip being the easiest, (100)[010] slip and a sluggish (010)[001] chain slip [118]. Values for critical resolved shear stress for those slips were not determined.

The stress induced martensitic transformation is a transformation from one crystallographic form to another form and associated by a displacement of chains to new positions in the new crystallographic cell in order to accommodate the deformation. An example of martensitic transformation from orthorhombic to monoclinic form was found in oriented polyethylene with well defined texture subjected to uniaxial compression. The martensitic transformation was also found in other polymers: in poly(L-lactic acid) [119] and in nylon 6 with the $\forall$ form transforming to the (form [120, 121].

iPP highly rich in $\beta$ crystal modification (92\%) were deformed by the plane-strain compression with constant true strain rate, at room temperature [122]. The results allowed to find out the deformation sequence and the active deformation mechanisms. The most important were interlamellar slip operating in the amorphous layers and resulting in localization of deformation in numerous fine deformation bands and the crystallographic slip systems, including the (110)[001] chain slip and (110)[1¹0] transverse slip. Deformation within deformation bands leads to $\beta \rightarrow$ smectic and $\beta \rightarrow \alpha$ solid state phase transformations. At room temperature the $\beta \rightarrow$ smectic transformation appeared the primary transformation and $\beta \rightarrow \alpha$ transformation yields only about $4 \mathrm{wt} . \%$ of new $\alpha$ phase at the same strain. With numerous fine deformation bands $\beta$-lamellae are locally destroyed and fragmented into smaller crystals. Another deformation mechanisms is a cooperative kinking of lamellae, leading to their reorientation and formation in a chevron-like lamellar arrangement. At high strains, above true strain equal 1, an advanced crystallographic slip and high stretch of amorphous material due to interlamellar shear bring further heavy fragmentation of lamellar crystals partially fragmented earlier by deformation bands. This fragmentation is followed by fast rotation of small unconstrained crystallites with chain axis towards FD. This process leads to development of the final texture of the highly deformed $\beta$-iPP with molecular axis of both crystalline and smectic phase oriented along the direction of flow. At higher 
temperatures in the range $55-100^{\circ} \mathrm{C}$ the most important deformation mechanisms found were interlamellar slip operating in the amorphous layers, resulting again in numerous fine deformation bands and the crystallographic slip systems, including the (110)[001] $\beta$ chain slip and (110) $\left[1^{-10}\right] \beta$ transverse slip. Shear within deformation bands leads to $\beta \rightarrow \alpha$ solid state phase transformation in contrast to $\beta-\rightarrow$ smectic transformation observed at room temperature. Newly formed $\alpha$ crystallites deform with an advancing strain by crystallographic slip mechanism, primarily the (010)[001] a chain slip. As a result of deformation and phase transformations within deformation bands $\beta$ lamellae are locally destroyed and fragmented into smaller crystals. Deformation to high strains, above true strain equal 1, brings further heavy fragmentation of lamellae, followed by fast rotation of crystallites with chain axis towards the direction of flow FD. This process, together with still active crystallographic slip, leads to the final texture with molecular axis of both crystalline $\beta$ and $\alpha$ phase oriented along flow direction.

Another example of active martensitic transformation is $\gamma$ modification of polypropylene. Morphology and deformation behavior of iPP homopolymer containing exclusively $\gamma$ modification with only minor traces of $\alpha$ crystals, obtained by isothermal crystallization at high pressure of $200 \mathrm{MPa}$, were investigated by Lezak et al. [123]. Deformation experiments performed in the plane-strain and uniaxial compression, demonstrated higher modulus, higher yield stress and flow stress, yet slightly lower ultimate strain of $\gamma$-iPP as compared to $\alpha$-iPP. During plastic deformation numerous fine shear bands, initiated by the interlamellar shear of the amorphous layers start to develop already at the yield point. Their propagation across the sample causes a limited destruction of lamellae oriented perpendicularly to the direction of the band. Destroyed fragments of crystallites transform partially into smectic phase. No $\gamma-\alpha$ phase transformation was detected. With increasing strain the shear bands multiply and tilt towards the flow direction. Lamellae, already fragmented within shear bands, undergo kinking and rotation, resulting in the formation of a chevron-like lamellar morphology. Simultaneously, a relatively weak onecomponent crystalline texture is developed. This texture is described by the orientation of $\mathbf{c}$ crystallographic axis along CD, $\mathbf{b}$ axis $10-30^{\circ}$ away of LD towards FD and $\mathbf{a}$ axis $10-30^{\circ}$ away from FD. Both crystalline texture and lamellae orientation are developed due to the activity of the same deformation mechanism - the interlamellar slip produced by the shear within interlamellar amorphous layers. Activity of any crystallographic deformation mechanism within crystalline component, including the anticipated (001)[010] transverse slip, was not 
detected. The interlamellar amorphous shear appears the primary deformation mechanism of $\gamma$-iPP. The other identified mechanism, $\gamma$-smectic phase transformation, plays rather a minor, supplementary role in the deformation sequence. Plastic deformation behavior of iPP homopolymer crystallized exclusively in the $\gamma$ modification at higher temperature in the range $55-100^{\circ} \mathrm{C}$ was also studied by Lezak et al.[124]. During plastic deformation numerous fine shear bands, initiated by the interlamellar shear of the amorphous layers, start to develop already at the yield point. Their propagation across the sample causes a limited destruction of $\gamma$ lamellae oriented perpendicularly to the direction of the band. Destroyed fragments of crystallites partially reconstruct into either mesophase (smectic) domains or crystals of $\alpha$ phase, depending on the deformation temperature. Mesophase is produced upon deformation at room temperature, while at $55^{\circ} \mathrm{C}$ and above the crystalline $\alpha$ phase is formed instead. With increasing strain shear bands multiply and tilt toward the flow direction. Fragmented lamellae undergo kinking and rotation, which results in the formation of a chevron-like lamellar morphology. This leads also to a development of a weak crystalline texture. Both crystalline texture and lamellae orientation emerge due to the same deformation mechanism of interlamellar slip, produced by the shear within interlamellar amorphous layers. The activity of any crystallographic deformation mechanism within the crystalline component was not detected at any temperature. The interlamellar amorphous shear appears to be the primary deformation mechanism of $\gamma$-iPP. The other identified mechanisms, i.e., $\gamma$-smectic and $\gamma-\alpha$ transformations, play a supplementary role in the deformation sequence.

Twinning may occur in crystals of sufficiently low symmetry: cubic symmetry excludes twinning while orthogonal symmetry allows for twinning. Hexagonal crystal structure allows for twinning of low molecular weight materials while in polymer crystals of hexagonal symmetry the basic twinning plane would be perpendicular to chains and therefore forbidden. Twinning along other planes in hexagonal crystals is not possible because of their high symmetry. In polyethylene of orthorhombic crystal symmetry the twinning is expected along (110) and (310) planes [125]. Only (110) plane twinning was found in bulk polyethylene. Twinning along (310) plane is blocked in bulk polyethylene because the fold plane is the (110) plane. In contrast, in rolling in a channel at a high rate and to a high compression ratio the texture of HDPE sample consist of two components [126]. One of them is the (100)[001] component, while the two others are rotated by $\pm 53^{\circ}$ around the rolling direction coinciding with the position of (310) poles clearly indicating the $\{310\}$ twinning of the basic (100)[001] component. The twinning occurs on unloading, when the sample leaves the deformation zone between the rolls. The partial recovery of the strain produces a tensile 
stress along direction of loading. Twinning is activated at high strain rates because the sample does not have sufficient time for stress relaxation while at high compression ratio the material is highly oriented and contains no more folds in (110) planes. It was estimated earlier that the critical resolved shear stress in the twin plane to activate twinning is around $14 \mathrm{MPa}$ [127]. Therefore the tensile stress generated along LD on unloading must be at least 28MPa. Such stress is apparently generated on unloading only when high deformation rate is applied during deformation.

Besides polyethylene the twinning was found only in few polymers including isotactic polypropylene: twinning along (110) plane [114].

Stress induced martensitic transformation and twinning alone are not responsible for large strain deformation.

From the presented review of mechanisms of plastic deformation of amorphous and crystalline phases it follows that the easiest is the deformation of the amorphous phase since it requires very little stress; the crystallographic mechanisms of plastic deformation need larger stresses. Therefore it is expected that first the amorphous phase is deformed and then crystallographic mechanisms are activated. An illustrative experimental evidence of this prediction was presented in ref.[128] where the Hermans orientation parameters for the amorphous and crystalline phases of a series of tensile deformed isotactic polypropylene samples are mapped out. The data points are based on the measurements of birefringence, infrared dichroism and wide angle x-ray diffraction of samples fixed in a frame at constant length. At first the amorphous phase becomes oriented while the crystalline phase remains very slightly oriented or even oriented in the transverse direction (the reason for such behaviour of polypropylene is the presence of cross-hatched lamellae). When the draw ratio is further increased the amorphous phase becomes almost entirely stretched out and then the crystalline phase begins to orient. Finally, at high draw ratio the macromolecular chain fragments embedded in both phases become highly oriented. Other reports based on a relaxed material after deformation indicate the contrary: amorphous phase orientation is lower than the orientation of crystalline phase e.g. [129].

It may be concluded that most of plastic deformation of both crystalline and amorphous phases occurs due to shear stresses and the shear contributes greatly to plastic deformation. It is then obvious that the great amount of plastic deformation is usually found at an acute angle with respect to applied tensile or compressive forces.

There are several ways of achieving plastic deformation of macroscopic samples. Those deformation methods in which some amount of hydrostatic pressure is generated in the 
material prevent for cavitation of the material. Because of the absence of cavitation the material undergoes plastic deformation via shear yielding; cavitation is damped. Also crazing is not preferred under hydrostatic pressure as it involves volume increase and the production of empty spaces between tufts.

In a macroscopic sample of a semicrystalline material subjected to stress few or all of the presented mechanisms of plastic deformation are orchestrated. Some of them are preferred, because of low shear stress required, and show up in early stages of deformation; others are activated in later stages under larger stress. The intensity of a particular mechanism may also change, for example, if the possibility of a certain slip is already exhausted or that the other slip mechanism rotated the crystals in such a way that the process mentioned is not now possible. As it was shown the mechanisms or their intensity may change while changing the temperature, pressure or the deformation rate. Together with the complicated aggregated supermolecular structure the process of the deformation in semicrystalline polymers is complicated and not easy to track.

\section{Dislocations in crystal plasticity}

In 1949 Frank [130,131] pointed out the possibility that the growth of crystals could take place because of the formation of dislocations in the crystal so that any real crystal should have a number of dislocations with a screw component, terminating on the face. When growth take place on these exposed molecular terraces, the edges of these layers develop into spirals centered on the dislocation. The theory of crystal growth based on dislocation theory as formulated by Frank [131] predicts the presence of growth features in the form of flat, spirally terraced hills on the crystal face which is perpendicular to a screw dislocation line. The height of a terrace should be just one unit of a crystallographic cell. In fact that was confirmed by multiple-beam interferometry of growth face of a beryl crystal. Crystallization of any substance is always easier, if screw dislocations are engaged, due to a dihedral angle benefit. The observed density of dislocations varies widely on different specimens and different low molecular weight materials, ranging from a few to $\sim 10^{4} / \mathrm{cm}^{2}$. It was found that on any crystal they are predominantly of one hand. Crystallization of polymers is also prompted by screw dislocations. Screw dislocation growth mechanism in polymers was reported since long time $[132,133]$ in polymer single crystals. In polymer crystals the estimate of the number of existing dislocations is few orders of magnitude larger $10^{5}-10^{8} / \mathrm{cm}^{2}$ than in low molecular substances. [134]. In terms of the introduction of screw dislocations, Schultz and Kinloch $[135,136]$ presented a model calculation of the twisting correlation of crystallites 
in a banded spherulite (a spherulite showing periodic extinction bands under polarizing optical microscope) with the row of screw dislocations of the same handedness. Based on their argument, Toda et al. proposed the possibility of the twisting correlation due to the introduction of the selective screw dislocations in the chair type PE crystals for the formation of banded spherulites of poly(vinylidene fluoride) [137]. Recently, Ikehara et al. [138] showed by 3D electron tomography, apparently searching for so-called "giant" screw dislocations, that no such screw dislocations are engaged in the formation of banded spherulites of PCL/PVB blends. Nevertheless, the existence and significant role of screw dislocations in melt crystallized polymers is well established [e.g. 139-141].

Screw dislocations observed in single crystals of polymers may have the Burgers vector extraordinarily large, up to $10 \mathrm{~nm}$ or more, being equal to the total thickness of lamellar crystals [134]. Such dislocations are easily detectable in microscopes, yet they do not have hollow cores as would be required of such large dislocations in most materials. In these lamellae the axes of giant screw dislocations are parallel to molecular stems. Such dislocations involve a readjustment in chain folding on a large scale in order to avoid formation of holes. As a consequence they are immobile. During crystallization they appear sporadically at growth fronts, especially at chain reentering. The giant screw dislocations play a vital role in lamellar branching and in morphological development of radiating polycrystalline aggregates. Spatial constraints arising during formation of giant dislocations stimulates adjacent lamellae to develop similar dislocations-the phenomenon often observed in stacks of lamellae. The role of giant dislocations in crystallization by chain folding was already recognized many years ago (see, for example, a review in Geil's book [132], also a discussion on dislocations in polymer crystals [142].

The amount of mobile dislocations that is present in polymer crystals is sufficient to initiate plastic deformation of polymer crystals. However, during crystallographic slips many more new screw dislocations are generated at crystal edges and propagate through the crystal. This process of emission of dislocations from the edges of the lamellae across the narrow faces that was initially proposed by Peterson $[143,144]$ explored further by Shadrake and Guiu [145] and more rigorously by Young [146] has now been widely accepted. [140, 141, 147-149]. The model of thermal nucleation of screw dislocations from Peterson [143,144] and Young $[150,151]$ has been shown to account fairly well for the plastic behavior of PE $[152,153]$ and polypropylene (PP) [154] and for the yield stress dependency on crystal thickness. Elastic line energy calculations indicate that nucleation of screw dislocations is more favorable than that of edge dislocations [155, 156]. Glide is also easier for the former [157]. It has been shown 
that screw dislocations parallel to the chain stems may be nucleated from the lateral surface of thin polymer crystal platelets upon coupled thermal and stress activation [143, 144, 158]. The activation volume of dislocation emission was determined from strain rate jump experiments during uniaxial compression of polyethylene [149]. The mean level of activation volumes were of the order of 350 of crystallographic unit cells and the estimated typical radius of the dislocation was $3.8 \mathrm{~nm}$, and much smaller indeed than the usual lamella thickness of $20 \mathrm{~nm}$ [159]. The Burgers vector of such dislocation must be small, of order of few lattice units only. Apparently the dislocations of such characteristics are the easiest to generate. It is then logical to assume similar values for the activity area of screw dislocations arising during melt crystallization.

The detailed knowledge concerns the system of crystallographic slips operating in polymer crystals, critical resolved shear stresses, twinning, martensitic transformation and succession of activation of individual slip mechanisms [111, 160, 161].

The magnitude of the shear stress needed to move a dislocation along the plane was first determined by Peierls [162] and Nabarro [163]. For orthorhombic unit cell of a crystal it varies exponentially with the ratio of both unit cell axes perpendicular to the macromolecular chain direction. For a (100) plane being the closed-packed plane (a axis larger than b axis) the shear stress reaches the minimum. That is because for closed-packed planes the interplanar bonds are weaker which results in lower activation energy and shear stress. The result is that the dislocations tend to move in the closest packed planes and in the closest packed direction because the Peierls-Nabarro force is smaller for dislocations with short Burgers vector.

\section{Generation of dislocations.}

As this mechanisms of dislocation propagation is widely accepted for slip mechanisms in semicrystalline polymers there are doubts about how the dislocations are generated. The density of dislocation in crystals is estimated at the level from $10^{5}$ to $10^{8} / \mathrm{cm}^{2}$ [164]. This number is not enough to give rise to a fine slip most often observed during polymer plastic deformation. There must be a way in which new dislocations are generated within crystals. In metals and other large crystals the identified source of dislocations is a multiplication mechanism known as Frank-Read source [165]. The Frank-Read mechanisms involves a dislocation line locked on both ends. When the shear stress is applied above a certain critical value the dislocation line will move to form first semicircle and then the line spiral around the two locked end forming finally the dislocation ring which will continue to grow outward 
under the applied stress. At the same time the original dislocation line has been regenerated and is now free to repeat the whole process. In this way a series of dislocation rings are generated indefinitely. In polymer crystals this elegant process is not active because the crystals are usually too thin for the dislocation line to spiral up and to form a dislocation loop.

Due to a peculiar structure of polymer crystals (long molecules which lay across the crystal thickness, fold and re-enter or take part in forming adjacent crystals) there are some restrictions which reduce the number of possible slip systems. Theoretical calculations demonstrated [166] and experiments confirmed [110] that polyethylene lamellae deform easily by slip in the direction of c-axis. There is a problem of the origin of the dislocations for activation of the slip mechanism. Shadrake and Guiu [166] pointed out that in the case of PE the energy necessary for creation a screw dislocation with the Burgers vector parallel to chain direction can be supplied by thermal fluctuations. It was shown that the change in the Gibbs free energy, $\Delta \mathrm{G}$, (ie. the energy which must be supplied by thermal fluctuations) associated with the creation of such dislocation under applied shear stress, $\tau$, is equal:

$\Delta G=\frac{K b^{2} l}{2 \pi} \ln \left(\frac{r}{r_{0}}\right)-\tau b l r$,

where 1 is the stem length; $b$ is the value of Burgers vector; $K$ is the shear modulus of a crystal; $r$ is the radius of dislocations (the distance from dislocation line to the edge of lamellae); and $\mathrm{r}_{0}$ is the core radius of dislocations.

There are still doubts if the model can be applied over the whole range of temperature i.e. from the temperature of glass transition to the onset of melting process. The dispute concerns the upper temperature of validity of this approach. Crist [167] suggested that the temperature of $\gamma$ and $\alpha$ relaxation processes of PE are the limits of applicability of the model. Young [103] and Darras and Seguela [168] have used that approach to model the yield behavior of bulk crystallized annealed polyethylene at a much higher temperature. Other authors [169] reported the existence of a transition in the range from $-60^{\circ} \mathrm{C}$ to $20^{\circ} \mathrm{C}$, depending on the material and strain rate, above which the Young's model can not be applied. They pointed out that there is a relationship between the transition temperature and $\beta$ - 
relaxation and suggested that below the transition temperature the yield process is nucleation controlled, while above propagation controlled. However, Galeski et al. [161] have shown that in plane strain compression for $\mathrm{HDPE}$ at $80^{\circ} \mathrm{C}$ the beginning of yielding is mainly associated with (100) chain slip within crystalline lamellae. Moreover, it was shown that for linear polyethylene only fine slip occurs below the deformation ratio of 3 associated with chain tilt and thinning of lamellae. Only at higher deformation the widespread fragmentation of those thinned to one third lamellae takes place. That is because further thinning becomes unstable- much like layered heterogeneous liquids responds by capillary waves and breakup of stacks of layers.

Seguela [170] proposed that the driving force for the nucleation and propagation of screw dislocations across the crystal width relies on chain twist defects that migrate along the chains stems and allow a step-by-step translation of the stems through the crystal thickness. The motion of such thermally activated defects is responsible for $\alpha$ crystalline relaxation.

When the problems mentioned above were investigated by others the crystal thickness of PE was controlled by changing the cooling rate, temperature of crystallization, using copolymers of PE characterized by different degrees of branching or crystallizing PE either from solution or from melt. The applied procedures allowed to obtain PE orthorhombic crystals with thickness over the range from $3 \mathrm{~nm}$ to $35 \mathrm{~nm}$. Crystallization of PE under elevated pressure evolved further by us [171] makes possible to obtain much thicker crystals. The method exploits the pseudo-hexagonal mobile phase of PE at certain range of pressure and temperature. The details how to obtain the samples with crystals of various thicknesses due to crystallization under high pressure are described in [171]. The great advantage of the approach is that the series of samples with various crystal thicknesses is obtained from the same polymer. Most of the previously reported studies of the structural changes caused by deformation were performed in a tensile mode, guided by obvious technological stimuli to 
explain processes associated with orientation by drawing. However, from the fundamental point of view the deformation by compression is more important. Uniaxial compression has the great advantage because the deformation is nearly a homogenous process and occurs without any significant deformation instabilities such as necking and cavitation. In the reported study [172] the samples prepared by high pressure crystallization were characterized by crystal thickness covering the range from 20 up to $150 \mathrm{~nm}$. Figure 7 presents typical true stress-true strain curves obtained in uniaxial compression for HDPE with various crystal thickness and crystallinity uniaxially compressed at a room temperature.

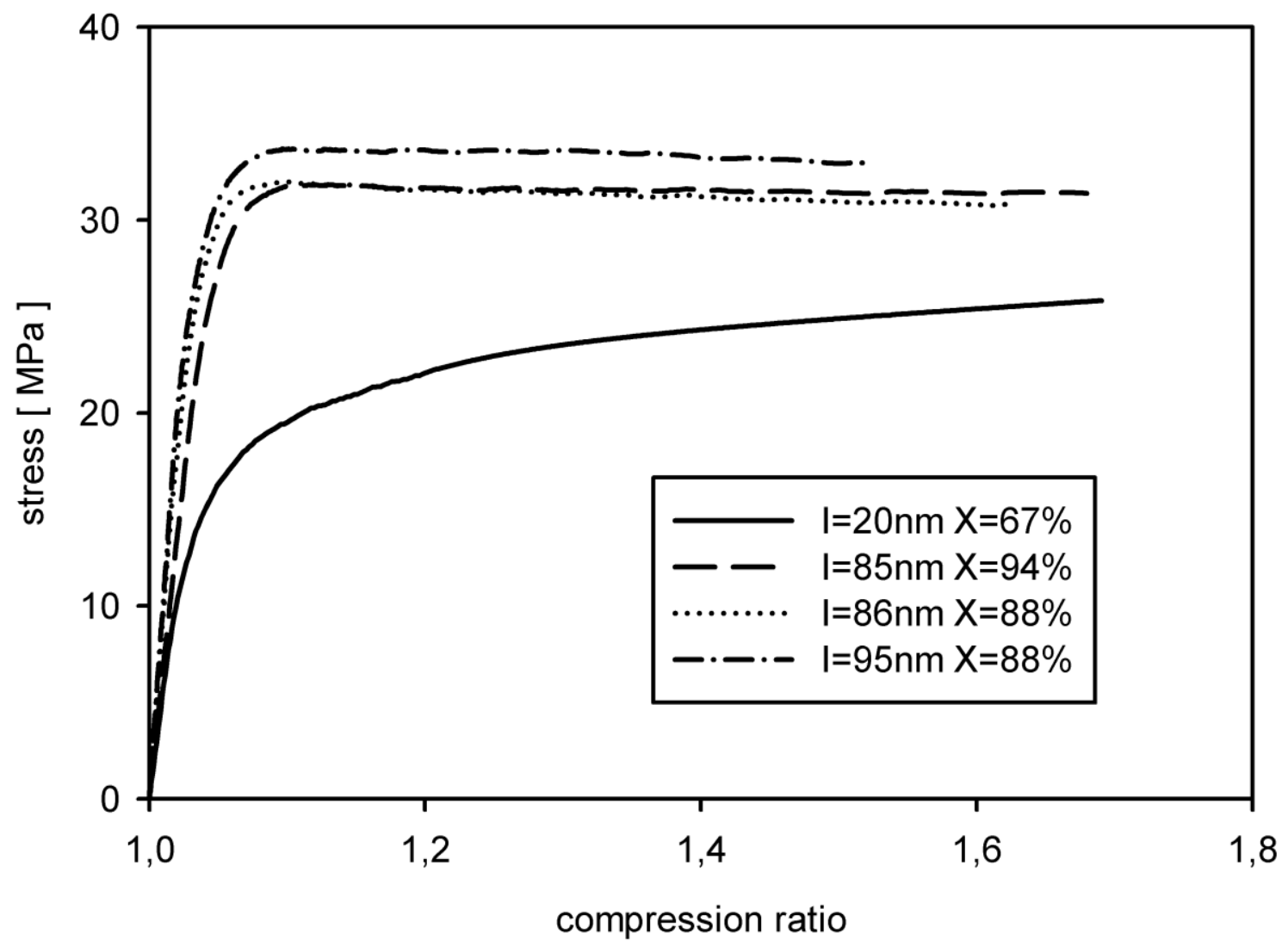

Figure 7. Stress vs. compression ratio for a series of HDPE samples differing in lamellae thickness and crystallinity. Uniaxial compression rate $0.000055 \mathrm{~s}^{-1}$.

After the usual initial elastic response below a compression ratio of 1.05-1.07 there is only a single yield and a region of intense plastic flow which sets in at a compression ratio of 1.12, followed by strain hardening. 
The changes in the yield stress with increasing crystal thickness are reported in Figure 8.

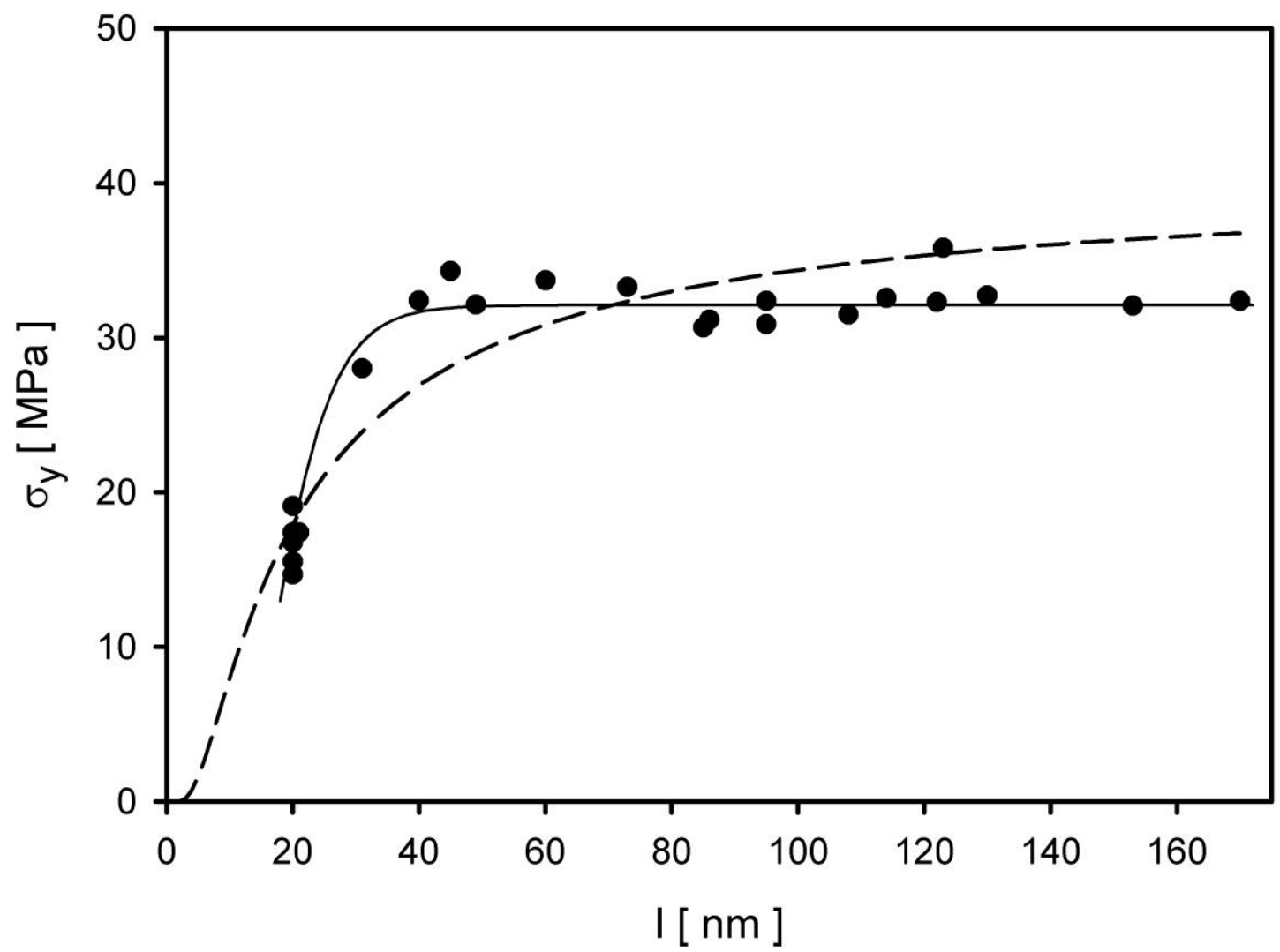

Figure 8. Yield stress in uniaxial compression plotted in relation to average lamellae thickness. The compression rate $0.000055 \mathrm{~s}^{-1}$. The dashed line is the best fit of eq.(3) from Shadrake and Guiu [166].

The yield stress increases with crystal thickness up to $40 \mathrm{~nm}$. This part of data agrees well with the published results of Brooks et al. [173] covering the range of lamellae thickness from 9.1 to 28.3. Beyond the region explored in the past the yield stress still increases in the range up to $40 \mathrm{~nm}$ and then, above $40 \mathrm{~nm}$, the dependence on crystal thickness abruptly saturates at the level of 29.5, 35 and $37 \mathrm{MPa}$ for initial compression rate of $0.000055,0.0011$ and $0.0055 \mathrm{~s}^{-1}$, respectively. The increase of the rate of compression increases the yield stress in the region of crystal thickness below $40 \mathrm{~nm}$ and also above 40nm. However, the saturation of the yield stress occurs at 40nm independently of the compression rate.

These observations imply that above 40nm the crystals thickness is no longer the decisive factor for the yield and that some other mechanism overtakes the control of the yielding process. Any "coarse slip" and other inhomogeneities in the course of compression which could decrease the yield stress did not occur. Instead as evidenced in SEM examination 
the chain tilt and homogeneous lamellae thinning occur up to the compression ratio above 2 (see Figure 9).

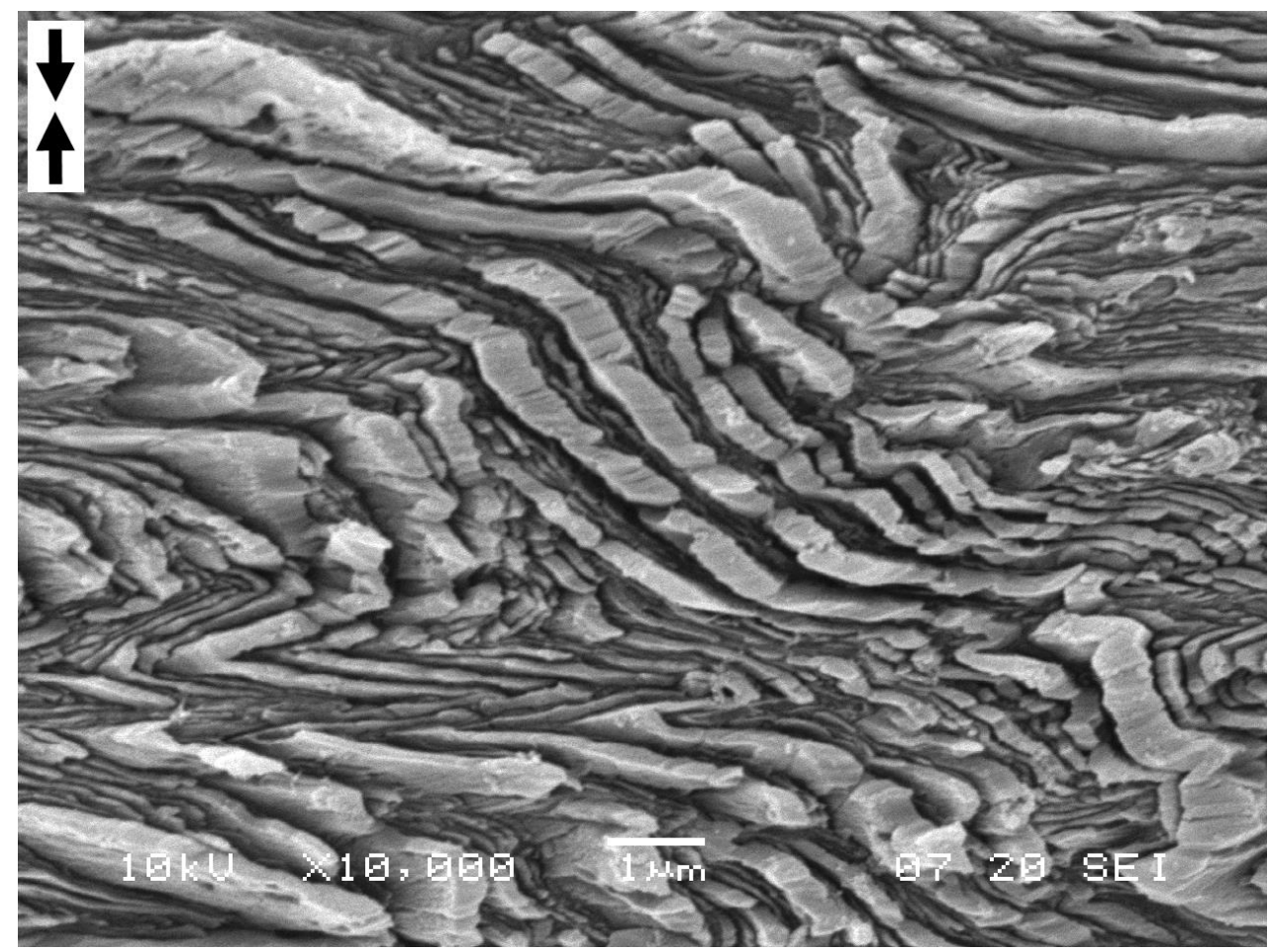

Figure 9. SEM micrograph of polyethylene uniaxially compressed to the compression ratio 3.175 with the rate $5.5^{*} 10^{-5} \mathrm{~s}^{-1}$. Polyethylene sample for compression test was prepared by crystallization under high pressure of $630 \mathrm{MPa}$ and temperature $235^{\circ} \mathrm{C}$. Initial average crystal thickness was $156 \mathrm{~nm}$ and crystallinity 87\%. Unpublished data connected with Ref. [149].

It can be concluded then that the observed yielding process is a "fine slip" process.

The overwhelming evidence accumulated over several decades is that the crystalline lamellae of PE, much like all crystalline ductile metals, deform plastically by the generation and motion of crystal dislocations and that while twinning and martensitic shears have also been suspected these do not make a substantial contribution. In coarse grained polycrystalline metals the rate mechanism of deformation is governed almost exclusively by either the intrinsic lattice resistance or the resistance of localized obstacles to dislocation motion. Dislocation nucleation in crystal plasticity as a rate controlling process is found only in nearly perfect crystals or in polycrystals in the nanoscale range. In PE and similar semi-crystalline polymers with chain molecules aligned nearly normal to the wide surfaces of the crystalline lamellae the preferred systems are those of chain slip on planes with the largest interplane separation: (100) in PE; (001) in nylon-6. In such systems because of the very high 
stiffness of the chain molecules it can be expected that the width of the core of the edge dislocations would be very large, resulting in only a negligible lattice resistance, making the screw dislocations with much narrower cores to be the most likely carriers of plasticity associated with the rate mechanism. That this had been so was confirmed indirectly by Peterman and Gleiter [174] who made the first TEM observations of screw dislocations in PE lamellae. The possibility that glide mobility of screw dislocations might be rate controlling was studied by Lin and Argon [72] in highly textured nylon-6 with quasi-single crystals where a generally convincing association was made to chain 'cross-overs' in the (001) planes as being the thermally penetrable obstacles to screw dislocation motion. Such chain 'cross overs' that are a topologically unavoidable product of large strain compression flow that was used to produce the quasi-single crystalline samples should, however, be largely absent in melt crystallized lamellae. For this and other reasons that the lamellae thicknesses are only in the nanoscale range $(10-20 \mathrm{~nm})$ it has been suspected for a long time that the rate controlling process must be repeated dislocation emission from lamellar edges under stress with the nucleated dislocations rapidly exiting the lamellae on the other side, requiring the process to repeat continuously. This process of emission of dislocations from the edges of the lamellae across the narrow faces that was initially proposed by Peterson [175] explored further by Shadrake and Guiu [168] and more rigorously by Young [176]. Screw dislocation model for yield in polyethylene] has now been widely accepted. It was re-examined in 2000 by Brooks and Mukhtar [173]. In the experiments of Kazmierczak, et al. [149] where PE with lamella thicknesses far exceeding the usual 10-20 nm (up to $170 \mathrm{~nm}$ ) were studied, it became clear that when lamella thicknesses exceed $40 \mathrm{~nm}$ the mechanism of emission of screw dislocation lines from lamella edges must be superseded by another, more ubiquitous one that is no longer sensitive to lamella thickness (compare Figure10). 


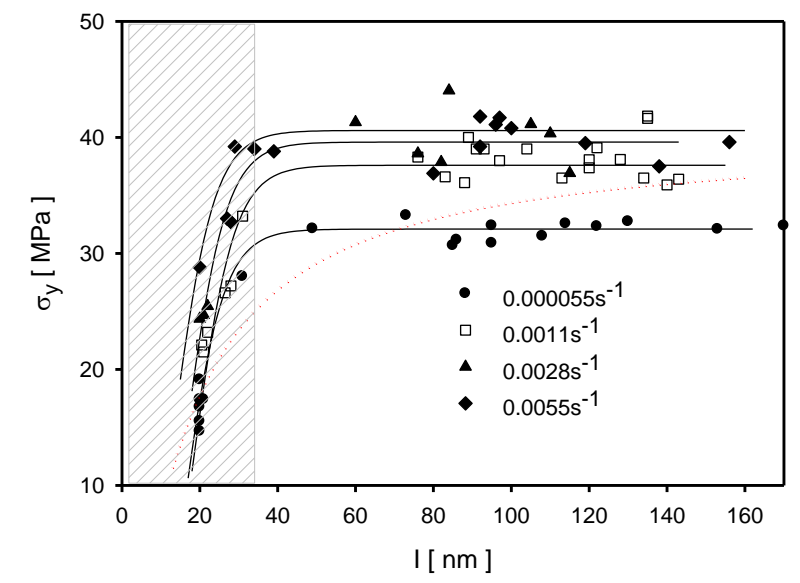

Figure 10. Yield stress of HDPE as a function of crystal thickness and compression rate. Redrawn from [149].

In examining new possibilities for the description of dislocation emission in PE crystals the recently developed fundamental considerations by Xu (Xu, et al. [177, 178], Xu and Zhang [179] and $\mathrm{Xu}[180])$ were revisited.

The large strain texturing deformation experiments and computer simulation of the entire process of texture-producing flow (Lee et al. [181], van Dommelen, et al. [182, 183] show that the contribution of the (100) [001] system to the total plastic deformation of PE is dominant and that its rate mechanism also dominates over all others. In the consideration of the rate mechanism of plastic flow in individual lamellae the attention is limited to deformations resulting from shear on the (100) [001] system as depicted in Figure 6. The principal local plastic deformation process is $\gamma_{13}$ shear, promoted by the resolved $\tau_{13}$ shear stresses, referred to the PE orthorhombic crystal structure, based on the well established slip on the (100)[001] crystallographic system [181-183]. 


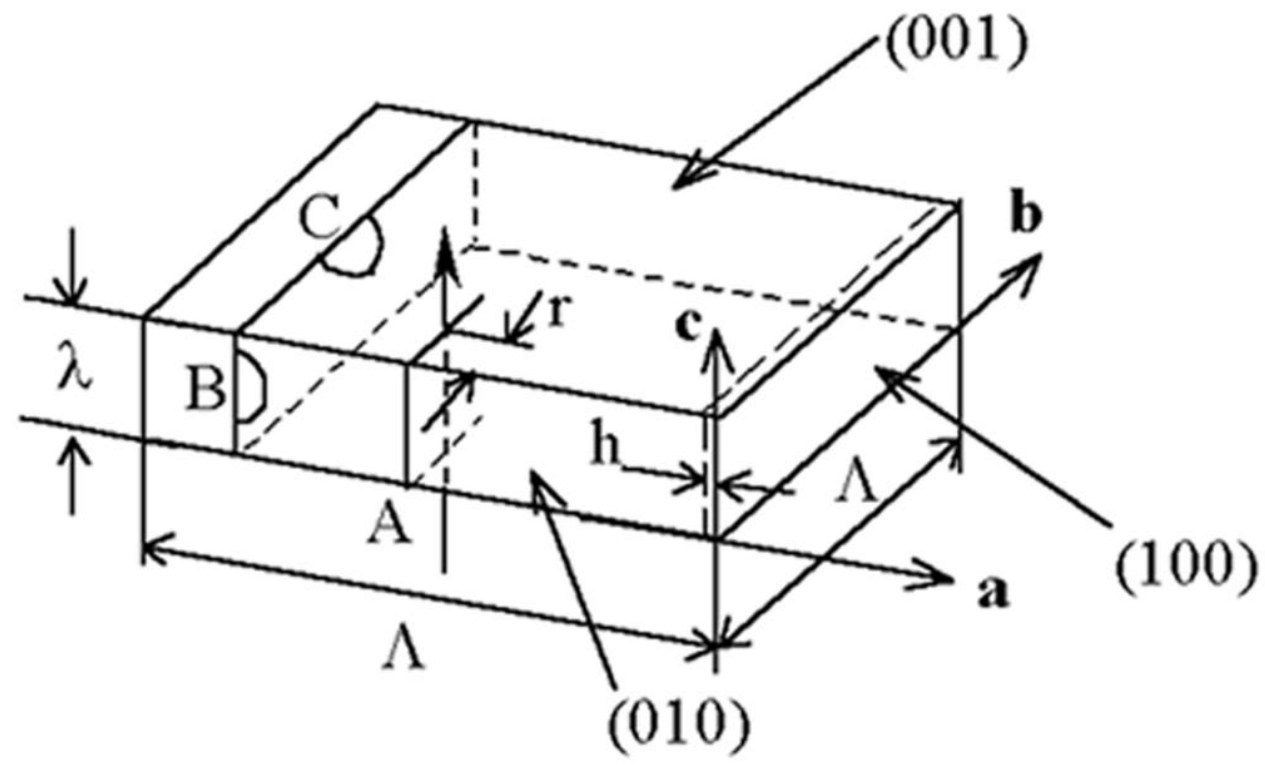

Figure 11. Sketch depicting a geometry of a typical lamella showing the principal chain slip system (100)[001] and the three separate modes of dislocation nucleation: $A$ monolithic screw, $B$ screw loop and $C$ edge loop.

Figure 11 depicts three modes of dislocation nucleation-controlled processes of chain slip on the (100) [001] system. The process identified as A is the previously considered one of nucleation of a fully formed screw dislocation monolithically from the narrow face. Clearly, as the lamella thickness 1 increases the ever increasing activation energy of this mode will no longer be kinetically possible. Thus, two other modes were considered: a screw dislocation half loop nucleation, still from the narrow face, and an alternative process of edge dislocation half loop nucleation from the wide face, depicted as processes $\mathrm{B}$ and $\mathrm{C}$, respectively, in Figure 11.

The energetics of all three processes has been considered rigorously by $\mathrm{Xu}$ and Zhang [179] and Xu [180]. In the paper by Argon et al. [159] the previous developments of screw dislocation emission monolithically from edges of thin lamellae were reconsidered and various questionable assumptions were eliminated. It was shown that when this is done the previously proposed model of screw dislocation emission from lamella edges can provide results as accurate as the more rigorous developments of $\mathrm{Xu}$, based on a variational boundary integral approach. In this case the Gibbs free energy change, $\Delta \mathrm{G}$, due to insertion of a fully formed screw dislocation from the edge is:

$$
\Delta G_{A}^{*}=\frac{\mu b^{3}}{4 \pi}\left(\frac{\lambda}{b}\right) \ln \left(\frac{1}{\beta}\right) \quad \beta=\frac{\tau}{\tau_{c}}
$$


where $\mu$ is the elastic modulus of the crystal, b is the Burgers vector of the lattice unit length, $\lambda$ is the lamellae thickness as defined in Figure $4, \tau_{\mathrm{c}}$ is the ideal shear strength of the (100) [001] chain slip system and $\tau$ is the acting shear stress. This formula is essentially similar to the formula derived by Shadrake and Guiu [166] although the fundamental parameters are expressed here in terms of shear strength of the slip system in exchange to two parameters: the radius of dislocations and their core radius. Both show a linear dependence of $\Delta \mathrm{G}$ on lamellae thickness.

The two other alternatives $\mathrm{B}$ and $\mathrm{C}$ where the lamella thickness plays no role were also considered by Argon et al.[159] leading to the following expressions:

Case B:

$\Delta G_{B}^{*}=\mu b^{3} g_{B}(\beta) \quad g_{B}(\beta)=\left(1-\beta^{2 / 3}\right) / \beta^{125}$

\section{Case C:}

$\Delta G_{C}^{*}=\left(\mu b^{3} /(1-v)\right) g_{c}(\beta) \quad g_{c}(\beta)=\left(1-\beta^{1 / 3}\right) / \beta^{1.15}$

A series of considerations including a correct value of $\mu$ modulus and its dependence on temperature, the kinetic crystallographic shear strain rate expression encountered in crystal plasticity, and a Coulomb law where the shear resistance $\tau$ is dependent on the normal stress $\sigma_{\mathrm{n}}$ acting across the glide plane lead to detailed expressions for yield stresses for all three cases of monolithic, half loop screw and half loop edge dislocations engaged in plastic deformation. The comparison for compressive yield stress of all three mechanisms with experimental data of Kazmierczak et al. [149] is illustrated in Figure 12. 


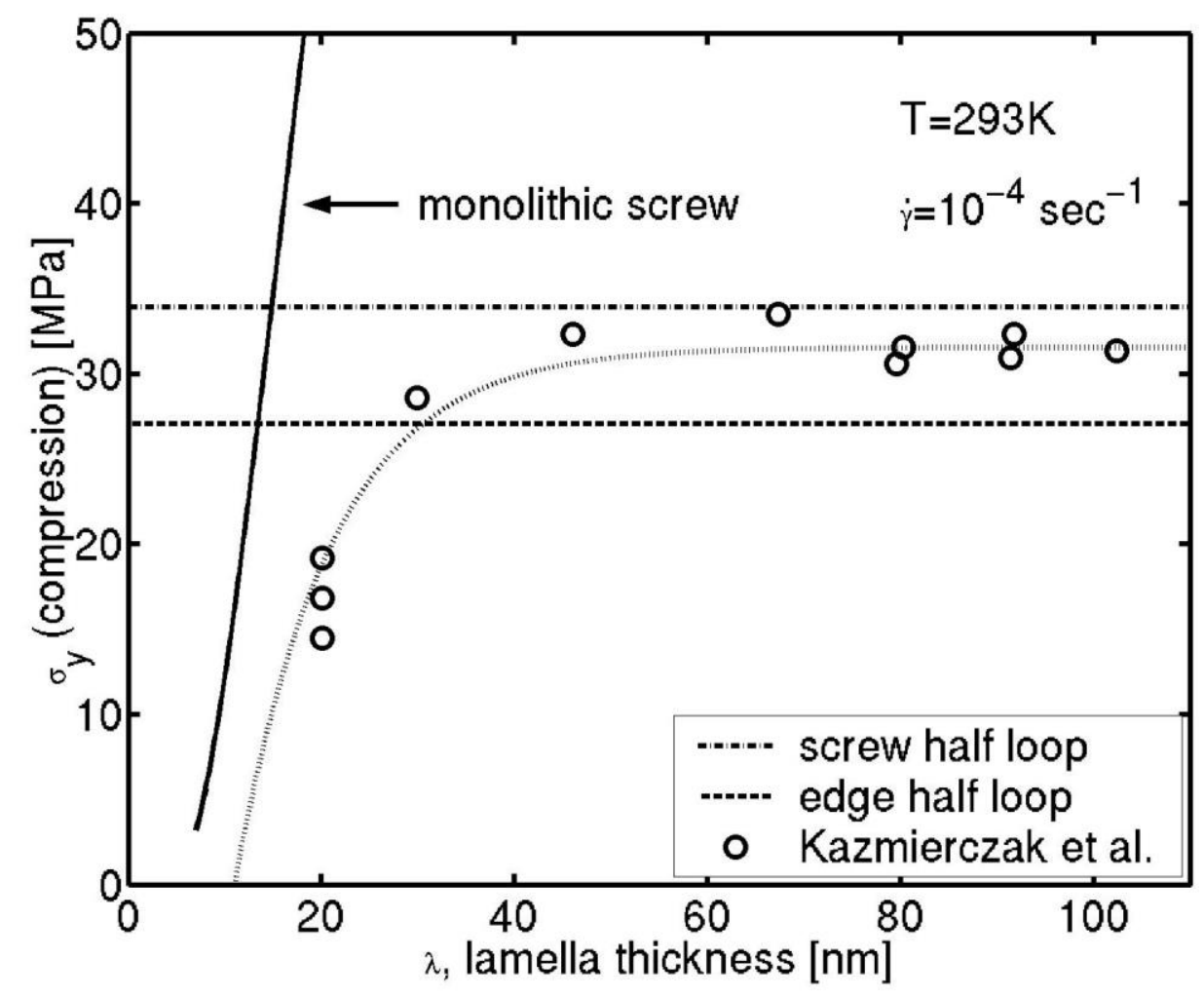

Figure 12. Dependence of compressive flow stress of polyethylene at $293 \mathrm{~K}$ and $\gamma^{-1}=10^{4} \mathrm{~s}^{-1}$ on lamella thickness: compared with three theoretical models. The data points from experimental work of Kazmierczak et al.[149]. Graph redrawn from [159].

The data points for thin lamellae are close to the prediction of the model of monolithic screw dislocations. The plot suggest a sharp departure from the mode of screw dislocation line nucleation to the half loop modes at roughly around $16 \mathrm{~nm}$ lamella thickness. The experiments show a more gradual transition at a lamella thickness of roughly $28 \mathrm{~nm}$. For lamellae thicker than $28 \mathrm{~nm}$ the data points fall in between the models for nucleation of edge half loops and screw half loops.

The results of experiments by Kazmierczak, et al. [149] with PE containing lamellae of much larger thicknesses than those of usual 10-15 nm have forced a re-examination of not only the model of Young, most recently considered again by Brooks and Mukhtar [173] but also other alternatives that must take over for thicker lamellae. These two new alternatives that are no longer dependent on lamellae thickness have explained well the leveling of the plastic resistance for lamellae of thicker than roughly $20 \mathrm{~nm}$. Also the new alternatives are in far better agreement with the published temperature dependences of the plastic resistance of PE than the previous model of Young (see Figure 8) and also predict well the measured levels on activation volume. 


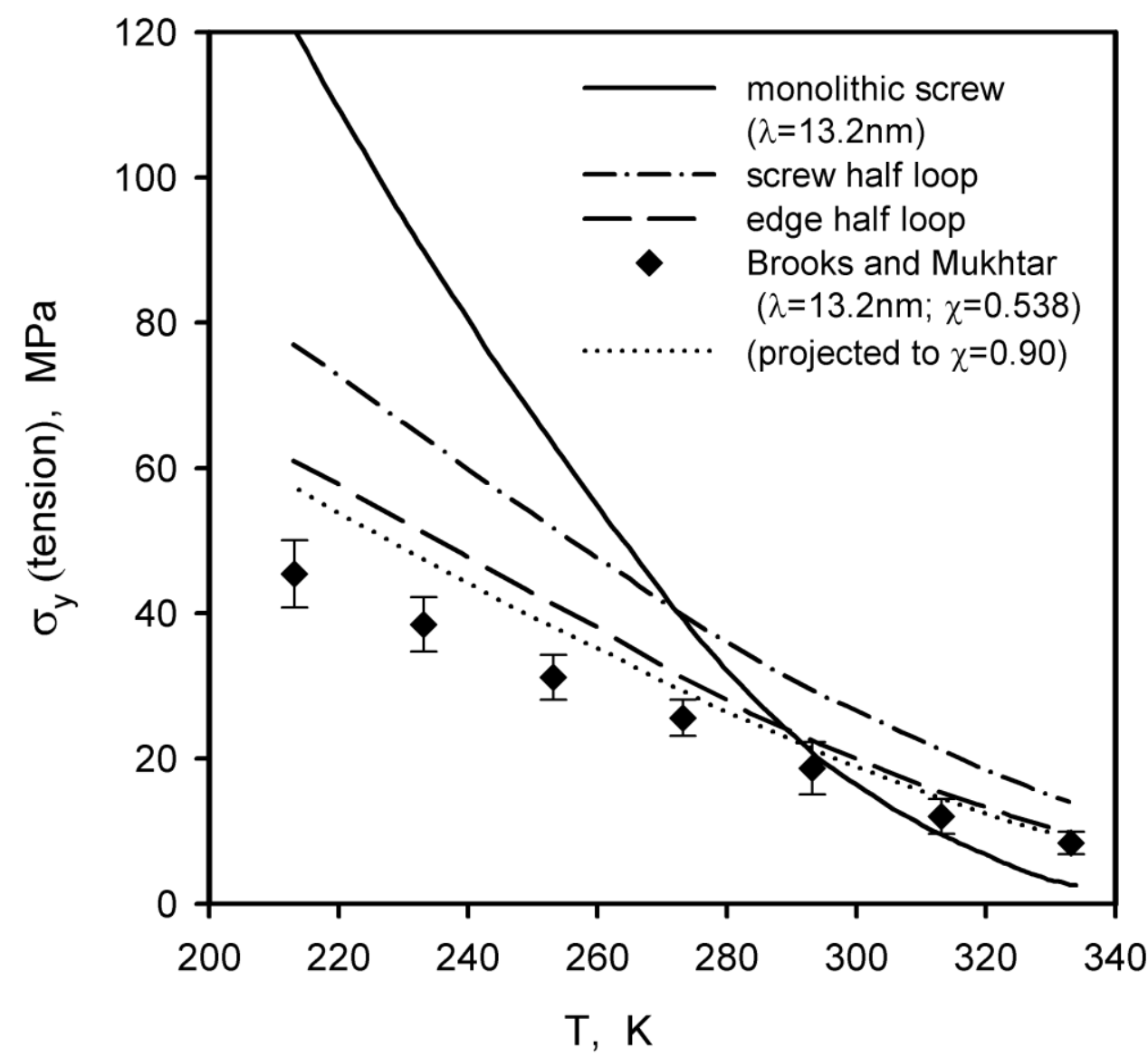

Figure 13. Predictions of modes A, B and C models of dislocation nucleations for the temperature dependence of the tensile yield stress of polyethylene compared with experimental results of Brooks and Mukhtar [173] for their PE2 material of moderate level of crystallinity at $X=0.538$. Dotted line projects experimental results for $X=0.9$.

The calculated temperature dependent tensile resistances are plotted in Figure 13 for the mode of monolithic screw dislocation emission from lamella edges and those of edge and screw half loop emissions from the large and small faces of the lamellae, respectively. The experimental data points of Brooks and Mukhtar [173] lie along a line that parallels the half loop nucleation modes and not the model of the usually considered mode of nucleation of monolithic screw dislocations. However, their positions are considerably below the models of the half loop nucleation. The discrepancy is partly due to the relatively low level of crystallinity of $\sim 0.538$. If the data were converted to account a crystallinity of 0.9 the experimental points would move up to the dotted curve lying much closer to the model of nucleation of edge dislocation half loops. We note that from the predictions of Figure 13, for thinner lamellae the flow stress might be governed by the mechanism of monolithic emissions of screw dislocations, the mechanism can switch over to that of half loop nucleation at lower temperatures. 
The kinetic mechanism governing dislocation controlled plasticity can be tested in a strain rate jump experiments, where during monotonic deformation, the strain rate $\mathrm{d} \gamma_{1} / \mathrm{dt}$ is abruptly increased or decreased to $\mathrm{d} \gamma_{2} / \mathrm{dt}$ and the resulting stress jump $\Delta \sigma$ is recorded. The parameter:

$\Delta v^{*}=(\mathrm{kT} / \Delta \tau) \ln \left[\left(\mathrm{d} \gamma_{1} / \mathrm{dt}\right) /\left(\mathrm{d} \gamma_{2} / \mathrm{dt}\right)\right]$

is related to the activation volume $\Delta v^{*}$ that characterizes the dependence of the activation free energy on shear stress. In equation (6), $\Delta \tau$ must be the shear stress change on the glide system. This is related by factor of 3 to $\Delta \sigma$ which is recorded in a uniaxial experiment. The measurement of $\Delta v^{*}$ allows for classification of material response to strain rate change, and in this case serves to identify the rate controlling mechanisms. We note here that experimentally in an uniaxial straining experiment when a strain rate change is made the change observed is in the uniaxial flow stress $\Delta \sigma$, while the definition of the activation volume must be related to the active shear mechanism and the change $\Delta \tau$ in shear resistance in spherulitic PE, ie, $\Delta \sigma=3 \Delta \tau$, similar to the relation in a polycrystalline metal [72].

The resulting response of a positive strain rate jump during monotonic deformation in positive strain rate jumps a small yield phenomenon is developed when the sample with thick crystals are deformed and only very small yield phenomenon for thin crystals the activation volume increases with the increase of crystal thickness and then stabilizes for thicker crystals, i.e. the changes of activation volume follow the same trend as the yield stress with the increase of crystal thickness which saturates for crystals thicker than $40 \mathrm{~nm}$.

\section{Competition between crystal plasticity and cavitation}

Crystalline polymers are often characterized by two parameters: long period based mostly on SAXS measurements and the crystallinity degree based on WAXS, DSC, NMR and other combined techniques. There is a large spectrum of long period values from $8.0 \mathrm{~nm}$ for PA 6 to 25-30 nm for HDPE. Also the crystallinities are varying substancially: from 5-10\% for olefin copolymers, through $20-30 \%$ for low density polyethylenes, $35 \%$ for PA 6 to 60 $70 \%$ for HDPE and POM. Comparison of these data with mechanical properties leads to the conclusion that polymers with higher crystallinity and thicker long period, e.g. POM, PP, HDPE are those for which large differences in tensile drawing and plane strain compression responses are visible. The exception from this scheme is PA 6, which is not very crystalline, 
having low long period, but characterized by high melting temperature. It suggests that its crystals are rather strong, exhibiting high resistance to shear. In fact polyamide 6 exhibits high critical shear stress for crystallographic slips [72].

The differences in tension/channel die compression tests are the largest in those polymers in which the crystals are more rigid and resistant to plastic deformation, such as in POM, PA 6, PP and HDPE. The level of stress necessary to achieve plastic deformation of crystals in the second group of materials is much smaller than in the first group. Negative pressure generated at concentration sites under such stress is then lower than the negative pressure needed to cause cavitation (below -20 MPa). In compression the yield stress reflects the plastic resistance of crystals. The immediate conclusion can be deduced that the cavitation during drawing can be observed only in polymers in which the value of stress at yield in compression (the factual yield stress for crystal plasticity) generates the pressure higher than the negative pressure required for cavitation. In the other case a crystallographic slip will occur earlier relaxing the stress and cavitational pores will not appear. This necessary condition for cavitation can be written in the form:

$\sigma_{\text {yield }} / 3=-\mathrm{p}>-\mathrm{p}_{\mathrm{cav}}$

where $\sigma_{\text {yield }}$ is the yield stress in compression corrected for stress sensitivity factor $\mathrm{K}$ as followed from Coulomb criterion, $\mathrm{p}$ is the negative pressure generated in channel die compression at yield and $\mathrm{p}_{\mathrm{cav}}$ is the negative pressure required for cavitation. In the other case a crystallographic slip will occur earlier relaxing the stress, and cavitational pores will not appear. This necessary condition for the activation of crystallographic slips can be written in the form

$\sigma>2 \tau_{0} /(1-K)$

where $\tau_{0}$ is the critical resolved shear stress for the easiest crystallographic slip.

From the above condition it follows that cavitation during drawing can be expected in such polymers as nylons (the easiest slip for PA 6 is $16.24 \mathrm{MPa}$ [72], polypropylene ( (010)[001] slip at around 22-25 MPa [184, 185], poly(methylene oxide) [186]. Only if thick crystals are present, cavitation can be present in polyethylene, since only thick PE crystals exhibit high enough yield stress in compression $[173,187,188]$. No cavitation is expected during deformation of low density polyethylene and quenched high density polyethylene, both 
having usually thin lamellar crystals, but cavitation can be found in HDPE which is slowly cooled when forming thick wall products.

It follows that in drawing there is a competition between cavitation and activation of crystal plasticity: easier phenomena occur first, cavitation in polymers with crystals of higher plastic resistance, and plastic deformation of crystals in polymers with crystals of lower plastic resistance.

\section{Micromechanics modelling in semi-crystalline polymers}

\subsection{Microstructure and mechanical properties}

At a nanoscale, the semicrystalline polymers can be seen as lamellar crystals, which are embedded by amorphous layers. This morphology imparts locally a high mechanical anisotropy, especially when the amorphous phase is in the rubbery state, like for polyolefins. When crystallizing from the melt without any deformation and any strong thermal gradient, spherulitic textures are formed by the radial growth of lamellae from a nucleation point, with intervening spaces filled by subsidiary lamellae, the polymer chains being more or less perpendicular to the basal plane of lamellae. These spherulites, which diameter order of magnitude is about 1 to $10 \mu \mathrm{m}$, have in fact a quite complex microstructure [189]: they look like a wheat sheaf close to the nucleation point (Figure 14). It is generally claimed that the spatial distribution of lamellae inside a spherulite is nearly isotropic, the random assembly of spherulites at a macroscopic scale is surely isotropic.

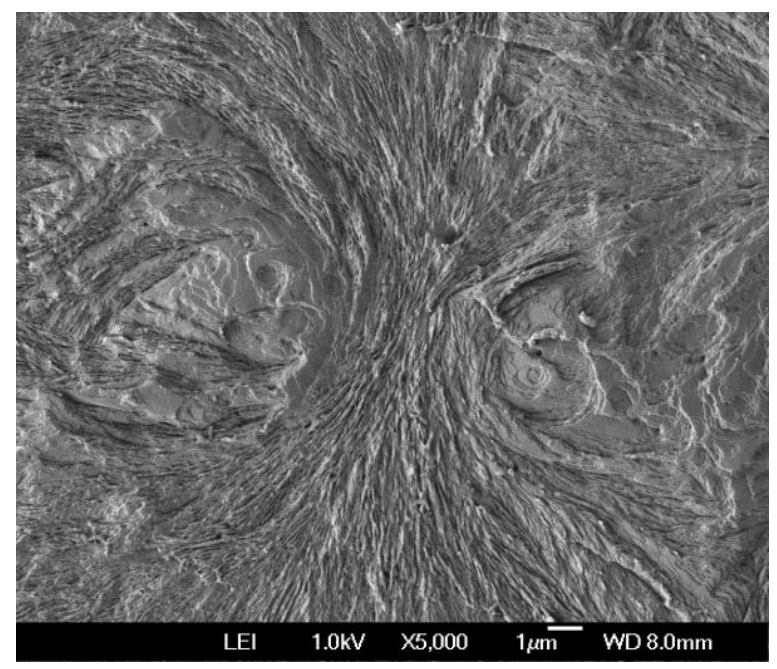

Fig. 14. Wheat sheaf form of a selective chemical 
From a mesoscopic point of view, when a semicrystalline polymer is submitted to a large deformation, the spherulitic structure is gradually broken down, while increasing molecular orientation. In a tensile deformation, the spherulitic could be reformed ultimately in a new oriented microstructure: the oriented fibrillar texture [190]. Such cold processed polymer fibers may have a young modulus, which could nearly reach $150 \mathrm{GPa}$ for polyaramid fibers $\left(\operatorname{Kevlar}^{\circledR}\right)$. Then, the strong link between microstructure and mechanical properties can be easily guessed.

When a semicrystalline polymer crystallises from the melt under a deformation, various crystalline superstructures appear: from deformed or oriented spherulites to very oriented structures like fibrils or shish-kebabs, which are considered as alternating amorphous and crystalline stacks growing around a fibril. The cooling of a melt is able to create these very oriented crystalline structures in polymers fibers for uniaxial elongation or PET bottles for biaxial elongation for example. But shearing can also generate very oriented crystalline microstructures [191]. The molecular orientation in the melt, which is being cooled, generates an oriented crystallization and therefore, the global orientation in the solid polymer is generally higher than the one that existed in the sheared melt [192]. The crystalline molecular orientation seems to be governed by the strain rate in the melt and the cooling rate, which limits the chain relaxation [193]. The size of the induced crystalline entities depends on the nucleation density, which is directly linked to the melt orientation before crystallization [194]. Therefore, crystalline entities can vary from shish-kebabs of less than 100nm in the solidified layer during the filling of an injection molded part to isotropic spherulites of several microns in the core of the part.

Unlike amorphous polymers, high variation and anisotropy of mechanical properties can be obtained in polymer processing. In injection molding for example, these variations of mechanical properties (Figure 15) induce anisotropic shrinkages and warpages. Their prediction are wished by industrials to reduce tool developing time and cost, but a thermomechanical calculation is needed in which thermoelastic properties at least are needed. How the mechanical properties depend on crystallinity and crystalline orientation [195], amorphous phase orientation or even crystalline macrostructure? Can semicrystalline polymers be considered as composites at a nanoscale and as heterogeneous material at a mesoscopic scale? Could micromechanical modelling give an answer to these questions? 

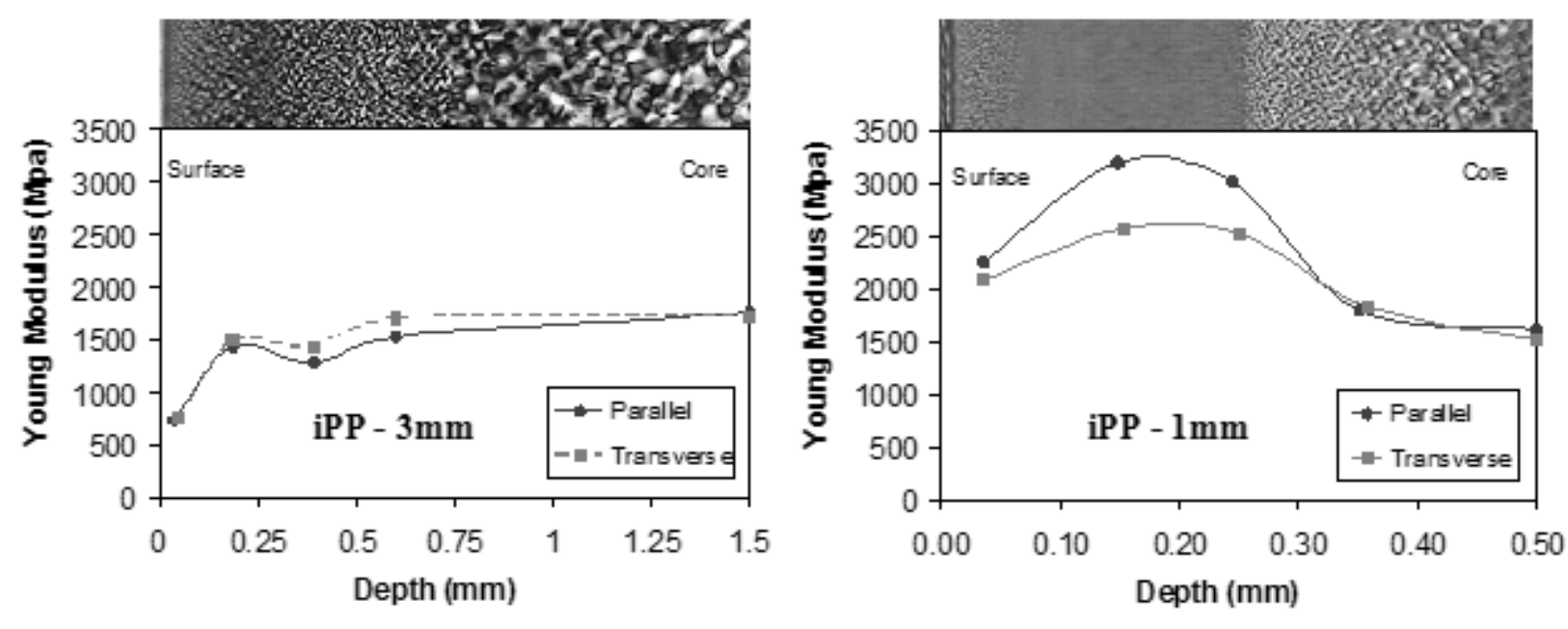

Fig.15. Young moduli versus depth of $1 \mathrm{~mm}$ - and $3 \mathrm{~mm}$-thick injection molded iPP plates measured in parallel and transverse directions to the flow: injection time $=1.6 \mathrm{~s}$, mold temperature $=40^{\circ} \mathrm{C}$. Superimposition of microscopic view under polarized light of the associated crystalline microstructure.

\subsection{The micromechanical models}

Considering a heterogeneous microstructure with known components properties, micromechanical modelling is aiming at establishing the behavior law of the equivalent homogeneous medium. Theoretical micromechanical modelling was subject of intensive studies with the outcome of composite materials and it has been applied with success for heterogeneous materials, such as metal alloys [196, 197].

Three main types of heterogeneous materials have been distinguished and treated:

a. Fillers surrounded by a matrix; this first family includes the polymers reinforced by mineral or organic fillers such as calcium carbonate particules [199] or glass fibers [200], carbon fibers and now nanofillers [201].The fillers are modelled by ellipsoids surrounded by a matrix and the two aspect ratios are the key parameters for such a representation.

b. Laminates. Stacks of unidirectional carbon fiber/epoxy composite sheet used in aeronautical industry for example are part of this family [202]

c. Aggregates of particles, which are generally one phase media, but because of random or partially random orientation of particles or crystals, discontinuities exist across interfaces. The metallic polycrystalline materials are typically members of this family [203].

When elastic behavior is only considered, the constitutive equations for the equivalent homogeneous medium may be written in terms of: 
where $\boldsymbol{\Sigma}$ and $\mathbf{E}$ denote the macro stress and macro strain tensor, respectively, $\mathbf{C}$ is the stiffness tensor of the semi-crystalline polymer. Introducing the representative volume element $V$ of the local properties, which contains a large number of micro-elements but has small dimensions in regard to the structure dimensions, $\boldsymbol{\Sigma}$ and $\mathbf{E}$ may be given in terms of the volume average stress and strain tensors:

$$
\boldsymbol{\Sigma}=<\boldsymbol{\sigma}>_{V}=\frac{1}{V} \int_{V} \boldsymbol{\sigma}(\mathbf{x}) d V \quad \mathbf{E}=<\boldsymbol{\varepsilon}>_{V}=\frac{1}{V} \int_{V} \boldsymbol{\varepsilon}(\mathbf{x}) d V
$$

$\boldsymbol{\sigma}$ and $\boldsymbol{\varepsilon}$ being the microscopic stress and microscopic strain tensors respectively. Due to Hill [204], strains (resp. stresses) in the heterogeneities are related to the macroscopic strains (resp. stresses) by the concentration-strain (resp. stress) tensorA:

$$
\boldsymbol{\varepsilon}^{c}(x)=\mathbf{A}: \mathbf{E} \quad \boldsymbol{\sigma}^{c}(x)=\mathbf{B}: \boldsymbol{\Sigma}
$$

The rigidity tensor $\mathbf{C}$ or the compliance tensor $\mathbf{S}$ of the homogenized material are simply defined by the relation:

$$
\mathbf{C}=\mathbf{C}^{a m}+f^{c}\left(\mathbf{C}^{c}-\mathbf{C}^{a m}\right) \mathbf{A} \quad \mathbf{S}=\mathbf{S}^{m}+f^{c}\left(\mathbf{S}^{c}-\mathbf{S}^{m}\right) \mathbf{B}
$$

where $f^{c}$ is the volume fraction of the crystalline phase. The assessment of the concentration-strain tensor A (or $\mathbf{B}$ ) depends on the micromechanical model. The simplest models are the Voigt ( $\mathbf{A}=\mathbf{I}, \mathbf{I}$ being the identity tensor) and Reuss $(\mathbf{B}=\mathbf{I})$ bounds, which correspond to a homogeneous deformation and homogeneous stress in the material respectively. Because of the high contrast between amorphous and crystalline phase moduli, both assumptions will give a result very far from the experiments. In another model due to Mori and Tanaka [205] the inclusions undergo the influence of other inclusions as equal as the average field in the matrix. This theory only applies for volume fractions up to $30 \%$. Crystalline phase volume fractions encountered in semicrystalline materials is out of the model validity range. The differential scheme is better designed when the volume fraction of the reinforcing phase is high [197]. Then, the reinforcing phase is introduced by infinitesimally small increments using the dilute-distribution assumption of Eshelby [206]. In the self consistent model, the material surrounding the inclusion is the equivalent homogeneous medium. This non explicit model was intensively used for all types of 
materials, but it is better suited for polycrystals or aggregates of inclusions than inclusions embedded in a matrix [207].

\subsection{Idealizing the microstructure of semicrystalline polymers}

Semicrystalline materials are often be considered as composite materials at a nanoscale and then the use of micromechanical models to predict the macroscopic properties are quite natural. But semicrystalline materials cannot be put so easily in one of the three classical previous modelled families defined for composites and polycrystalline materials because the crystalline microstructure is complex and organized at several scales. Then, the first question is: at which scale do we have to consider "this composite material"? At a microscale, that is the scale of the crystalline microstructure (spherulites, shich-kebabs)? Or at the nanoscale of the crystalline lamellae?

The microstructure is composed of spherulites when considering isotropic semicrystalline materials. Most of the works have found that the spherulite size has no significant effect or low effect on mechanical properties [208]. Then, the modelling at the nanoscale can be relevant in this case. It could be also true for very oriented polymers like fibers because the induced crystalline microstructure results mostly in microfibrils with sizes typically in the range $50-500 \mathrm{~nm}$, even if they are composed of some form of ordered crystalline and amorphous domain [209]. Between these two extreme cases, it seems more difficult to ignore the two scales. Few researchers applied micromechanics modelling to predict the macroscopic mechanical properties of semi-crystalline polymers, and nearly all the research works have only considered the nanoscale. Four general types of microstructure modelling have been performed.

The first attempts in the sixties and seventies proposed to predict elastic properties by using simple analytical models derived from the analysis of fiber-filled media. Takayanagi [210] described the elastic behavior of unidirectional oriented processed fibers. Halpin and Kardos [211] proposed to use Halpin-Tsai model [212]. Phillips and Patel [213] applied also this model to PE with an adjustable parameter linked to crystallite shape ratio. However, these models require the assumption that lamellae be regarded as fibers. Moreover, they are known to fit only the experimental data at low volume fraction of filler and this is not the case of semi-crystalline materials, for which the crystallinity can often reach 60 to $70 \%$.

Janzen [214] was one of the first to use the self-consistent model for semicrystalline polymers, but in its most simple form with spherical inclusions and isotropic media. The interaction between a lamella and the others is supposed to be given by the interaction of an 
inclusion with "an equivalent homogeneous medium" in which it is embedded and whose properties are those of the macroscopic material. A large collection of data on Young's modulus of unfilled and isotropic PE at ambient conditions has been compared with various competing theoretical mixing rules developed for composite micromechanics. It was found that the self-consistent scheme appears to have valid application to this system. The selfconsistent scheme with more elaborated constitutive laws for components was used by Parks and Ahzi [215] and Dahoun et al. [216] to predict the plastic behavior and the deformation texture of PEEK and iPP [217]. In all models, the amorphous phase behaviour is accounted for softening the macroscopic behaviour in an average sense.

Lee et al. [218, 219] defined a dedicated polymer micromechanical model in which the material is represented by an aggregate of layered two-phase composite inclusions (Figure 16). Large plastic deformation and texture evolution in initially isotropic high density polyethylene (HDPE) was simulated. This aggregate scheme was used later by Nikolov et al. [220] to model the viscoelastic behavior of semicrystalline polymers at small deformations and by van Dommelen et al. [183] to model the elasto-viscoplastic behavior of semicrystalline polymers under large deformations.

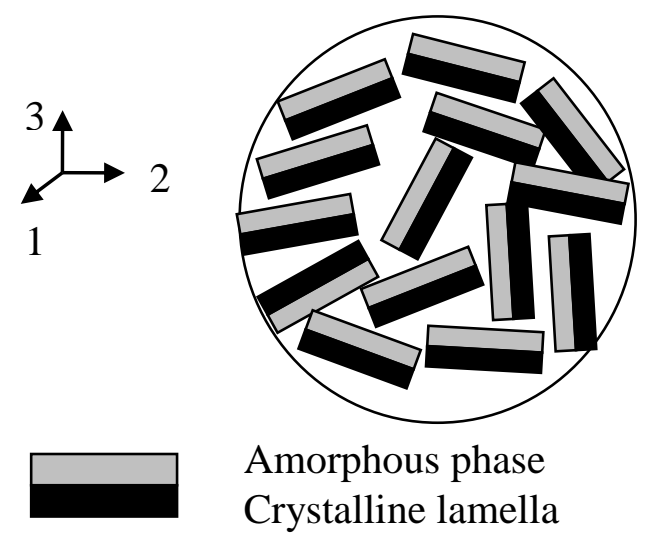

Figure 16. Aggregate of layered two-phase composite inclusions

More recently, Bedoui et al. [221, 222]. show that a classical inclusion / matrix model (Figure 17) associated with a differential scheme gives satisfactory results for predicting the elastic behavior of isotropic semicrystalline polymers. 


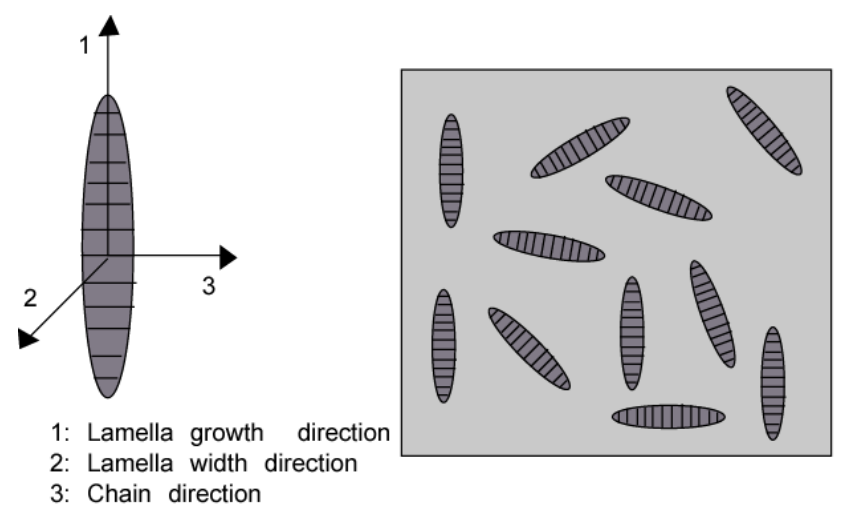

Figure 17. Schematic illustration of crystal lomallog mondomly distrihutad in tho

\subsection{Elastic behavior prediction}

For many applications however, the small-strain behavior is at least as important as the large deformations. As the prediction of elastic behavior of heterogeneous materials is the most known and well-established, it is worth to know if the current micromechanical models are able to predict the one of semicrystalline polymers. Firstly, the elastic properties of the composite components should be determined.

\subsubsection{Elastic properties of constitutive phases}

\section{Crystalline phase}

The crystalline phase consists of polymer lamellae, which show a highly anisotropic behavior with a very high modulus along the chain axis. Elastic constants have been theoretically calculated and experimentally determined for several polymers and are reviewed by Ward [190]. The rigidity tensors can be known with a good precision and three examples are given. Polymer chains, which have a helical conformation angle like PP [223] are less stiff in the chain direction. The theoretical rigidity tensor of PP $\alpha$-monoclinic crystal calculated by Tashiro et al. [224] gives a consistent value along the chain direction with experimental determinations of about $40 \mathrm{GPa}$ :

$$
\mathbf{C}_{P P}^{c}=\left(\begin{array}{cccccc}
7.78 & 3.91 & 3.72 & 0 & 0.9 & 0 \\
3.91 & 11.55 & 3.99 & 0 & -0.36 & 0 \\
3.72 & 3.99 & 42.44 & 0 & -0.57 & 0 \\
0 & 0 & 0 & 4.02 & 0 & -0.12 \\
0.9 & -0.36 & -0.57 & 0 & 3.1 & 0 \\
0 & 0 & 0 & -0.12 & 0 & 2.99
\end{array}\right) \quad G P a
$$


For orthorhombic form of PE or $\alpha$-form of PA, the theoretical values of the stiffness tensor seem to largely overestimate the measured ones [225]. The polyethylene chain has a planarzigzag conformation, which justifies a theoretical modulus as high as $300 \mathrm{MPa}$ along the chain direction. However dynamical calculations have demonstrated the high dependence of the modulus on the chain contraction and when the chain contracts slightly from the planarzigzag conformation, the modulus along the chain axis drops drastically. Tashiro et al. [223] suggest that, as the $\alpha$-form nylon- 6 , the PE polymer chain experiences a thermal motion at room temperature and contracts from the planar-zigzag conformation, which induces a drop in modulus. Therefore the moderate elastic stiffness tensor at ambient temperature could be, as proposed by Choy [225]:

$$
\mathbf{C}_{P E}^{c}=\left(\begin{array}{cccccc}
7.0 & 3.8 & 4.7 & 0 & 0 & 0 \\
3.8 & 7.0 & 3.8 & 0 & 0 & 0 \\
4.7 & 3.8 & 81 & 0 & 0 & 0 \\
0 & 0 & 0 & 1.6 & 0 & 0 \\
0 & 0 & 0 & 0 & 1.6 & 0 \\
0 & 0 & 0 & 0 & 0 & 1.6
\end{array}\right) G P a
$$

PET lamella stiffness was experimentally measured by Hine and Ward [226]. Then a theoretical estimate was provided by Rutledge [227]. For most components, experimental values of the elastic stiffness tensor are comparable to the theoretical ones. However, a discrepancy is also observed in the chain direction, which may be the result of imperfect crystallites due to chain axe misorientations. A better experimental estimate of this component has been given by Matsuo and Sawatari [228], and the PET crystal stiffness tensor is given by:

$$
\mathbf{C}_{\mathrm{PET}}^{\mathrm{c}}=\left(\begin{array}{cccccc}
7.70 & 5.46 & 5.07 & 0 & 0 & 0 \\
5.46 & 7.70 & 5.07 & 0 & 0 & 0 \\
5.07 & 5.07 & 118 . & 0 & 0 & 0 \\
0 & 0 & 0 & 1.62 & 0 & 0 \\
0 & 0 & 0 & 0 & 1.62 & 0 \\
0 & 0 & 0 & 0 & 0 & 1.12
\end{array}\right) \mathrm{GPa}
$$

\section{Amorphous phase}

In semicrystalline polymers, the amorphous phase is confined between crystalline lamellae and therefore the chain mobility should be reduced [229]. The confinement can also be seen as 
a phenomenon, which by increasing the density of entanglements, and so decreasing the molar mass between entanglements $M_{e}$, should increase the modulus.

When temperature is above glass transition, some authors $[183,214,220]$ have considered the possibility that the amorphous phase confinement does not significantly affect the rigidity of the amorphous phase and have considered it in a rubbery state. Other authors [230-233] chose a Young's modulus one or two orders of magnitude higher than the rubbery state. Their choice was generally not based on physics modelling or experimental evidence but on micromechanics modelling; the amorphous phase parameters are calculated by an inverse method and highly depend on the model used. Recently, Spitalsky and Bleda [234] have simulated the elastic properties of interlamellar tie molecules in semicrystalline polyethylene by atomistic modelling. In their work, it appears that the Young's modulus of PE tie chains only change drastically for highly extended chains. Considering the lack of experimental and theoretical evidences of a drastic change of the Young's modulus of the amorphous phase due to the crystalline environment, assuming the amorphous phase in a rubbery state when the temperature is above glass transition is not out of any sense. Then, chain entanglements are the cause of rubber-elastic properties and the kinetic theory of rubber elasticity, which was particularly developed by Flory [235], leads to the following equation:

$$
G_{N}^{0}=\frac{\rho R T}{M_{e}}
$$

For examples, $M_{e}$ can be chosen equal to $7 \mathrm{~kg} / \mathrm{mol}$ for PP and to $1.4 \mathrm{~kg} / \mathrm{mol}$ for PE [236], then the shear modulus $G_{N}^{0}$ at ambient temperature is equal to $1.5 \mathrm{MPa}$ for PE and 0.3 MPa for PP. Since the amorphous phase of the studied polymers is in the rubbery state, its Poisson ratio is very close to 0.5 , but slightly lower. To get coherent elastic properties, the Poisson ratio $v$ is calculated using the classical relation:

$$
B=\frac{E}{3(1-2 v)}
$$

where the bulk modulus $B$ can be determined from PVT data thanks to its definition relation:

$$
\frac{1}{B}=\frac{1}{V} \frac{\partial V}{\partial p}
$$

$V$ being the specific volume [221]. 
When temperature is under the amorphous glass transition, the physical phenomena are quite different and interactions between atoms occur at a much lower scale. Therefore, for small deformations the hypothesis of an elastic behavior of the confined amorphous phase close to the bulk one may be consider as a valid hypothesis.

\subsubsection{Some predictions of elasticity.}

Some very sophisticated models have already been performed to simulate the mechanical behavior of semi-crystalline polymers at large deformations, but could these models simulate at their limit the simple elastic behavior of isotropic semicrystalline polymers? Bedoui et al. $[221,222]$ have considered fully anisotropic crystallites embedded in a matrix and a layeredcomposite aggregate; several homogenization schemes have been used in those representations and the amorphous phase modulus varied. Finally only one model gives satisfactory trends and results for both modulus (Figure 18) and Poisson ratio (Figure 19): the crystalline lamellae embedded in a matrix associated with the differential scheme. The elongation ratio of crystalline lamellae was the only adjustable parameter, it was consistent with microscopic observations. However, the modelling is sensitive to the amorphous modulus and it is difficult to give a physical sense to the differential scheme.

Considering PET at ambient temperature that shows a low contrast in terms of elastic behavior between amorphous and crystalline phases, several models have been proven to provide similar results (Figure 20). In such a context, micromechanics models are only slightly sensitive to the crystallite shape ratio parameters. It explains why models with no crystallite shape ratio, like the U-inclusion model [183], still apply. Therefore, the obtained results in the case of glassy amorphous phases are contrasted. In terms of absolute values, the Young's modulus is decently approached by the models but in terms of the shape of the curve, the convexity of the experimental data is poorly represented. 


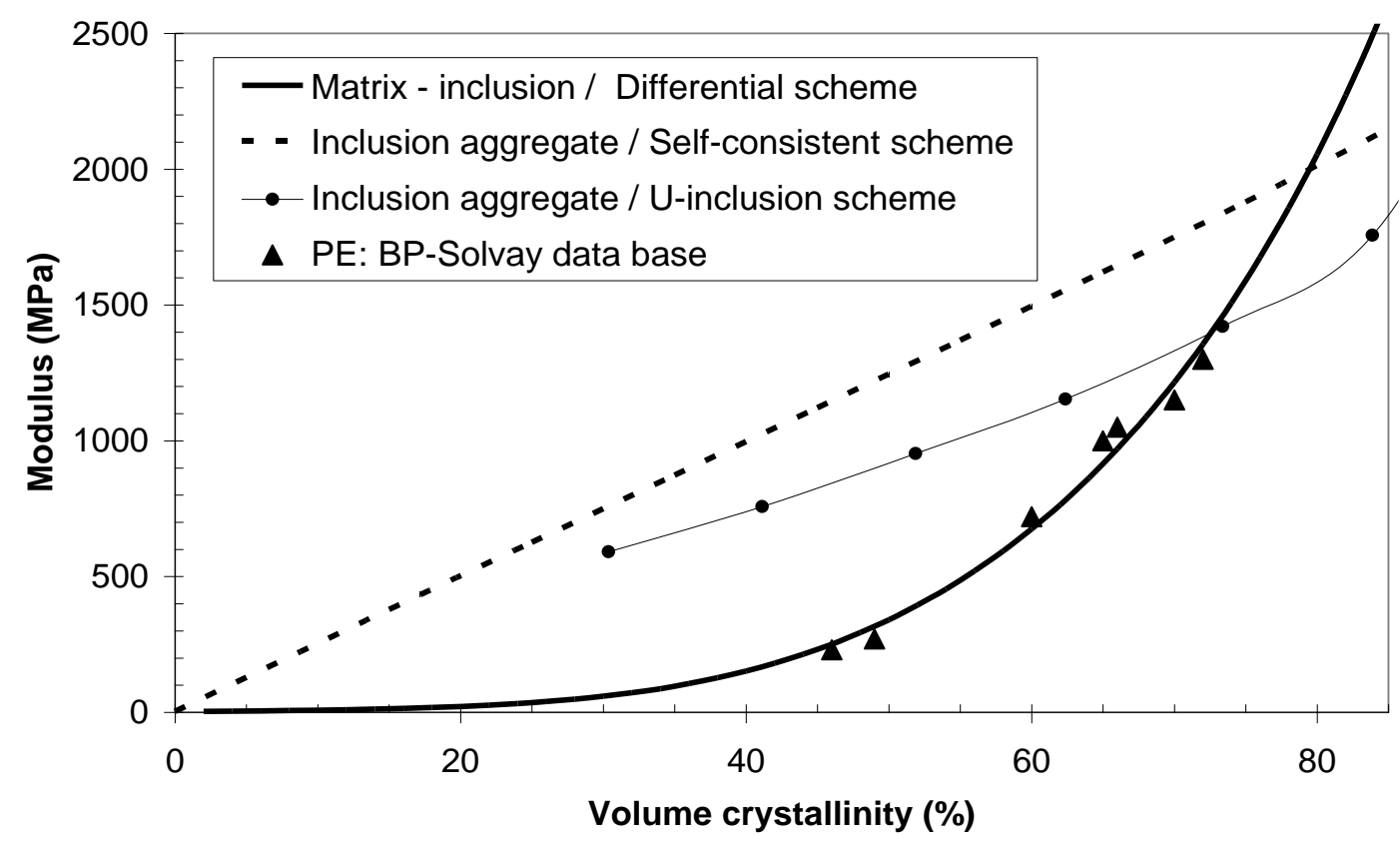

Figure 18. Prediction of macroscopic PE Young's modulus versus volume crystallinity. Ellipsoidal shape ratio $\mathrm{a}_{1} / \mathrm{a}_{3}=20$ and $\mathrm{a}_{2} / \mathrm{a}_{3}=5$ for matrix- inclusion scheme

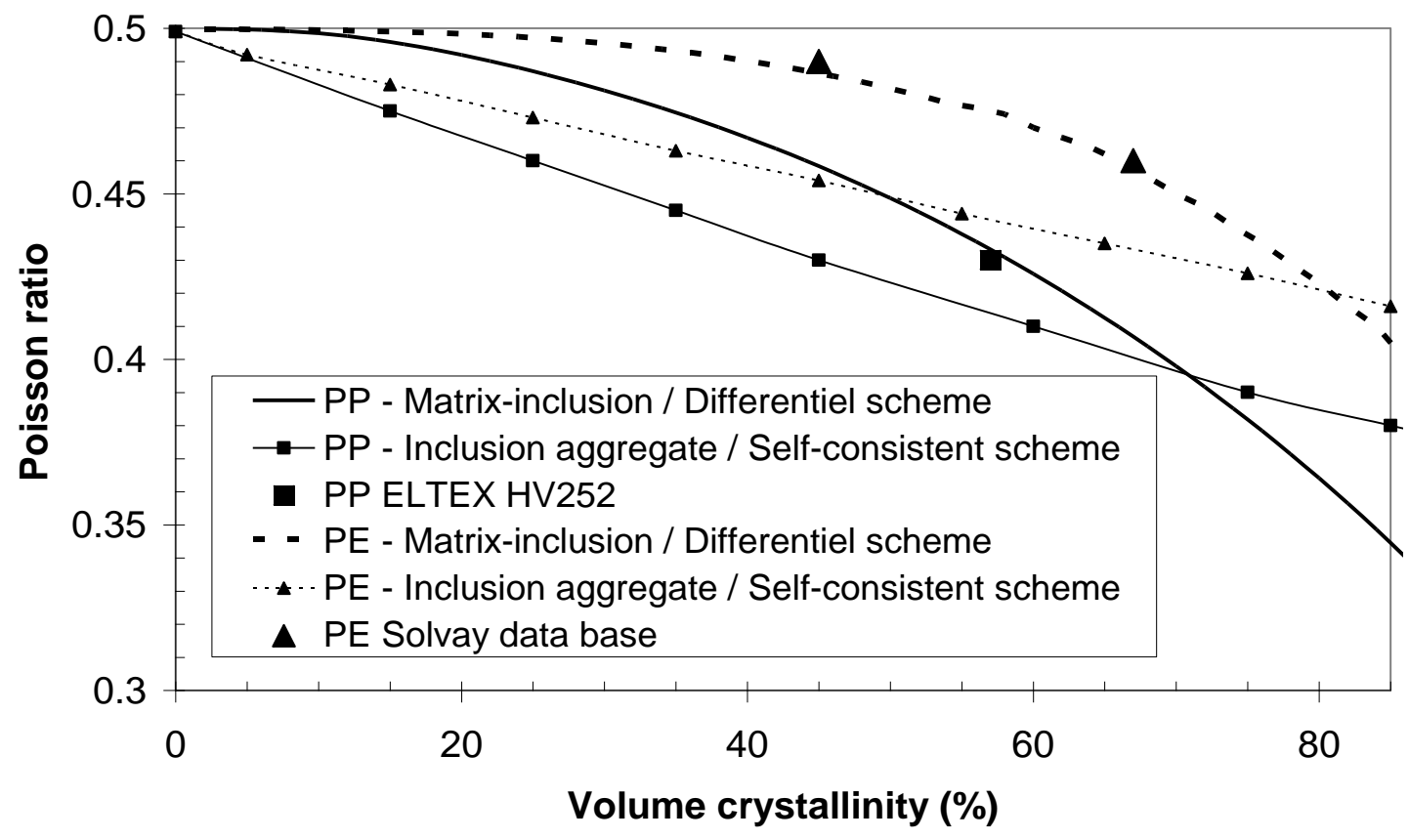

Figure 19. Estimates of PE and PP Poisson ratios versus volume crystallinity. Ellipsoidal shape ratios for matrix-inclusion model associated with the differential scheme: $a_{1} / a_{3}=20$ and $\mathrm{a}_{2} / \mathrm{a}_{3}=5$ for $\mathrm{PE}, \mathrm{a}_{1} / \mathrm{a}_{3}=100$ and $\mathrm{a}_{2} / \mathrm{a}_{3}=10$ for $\mathrm{PP}$. 


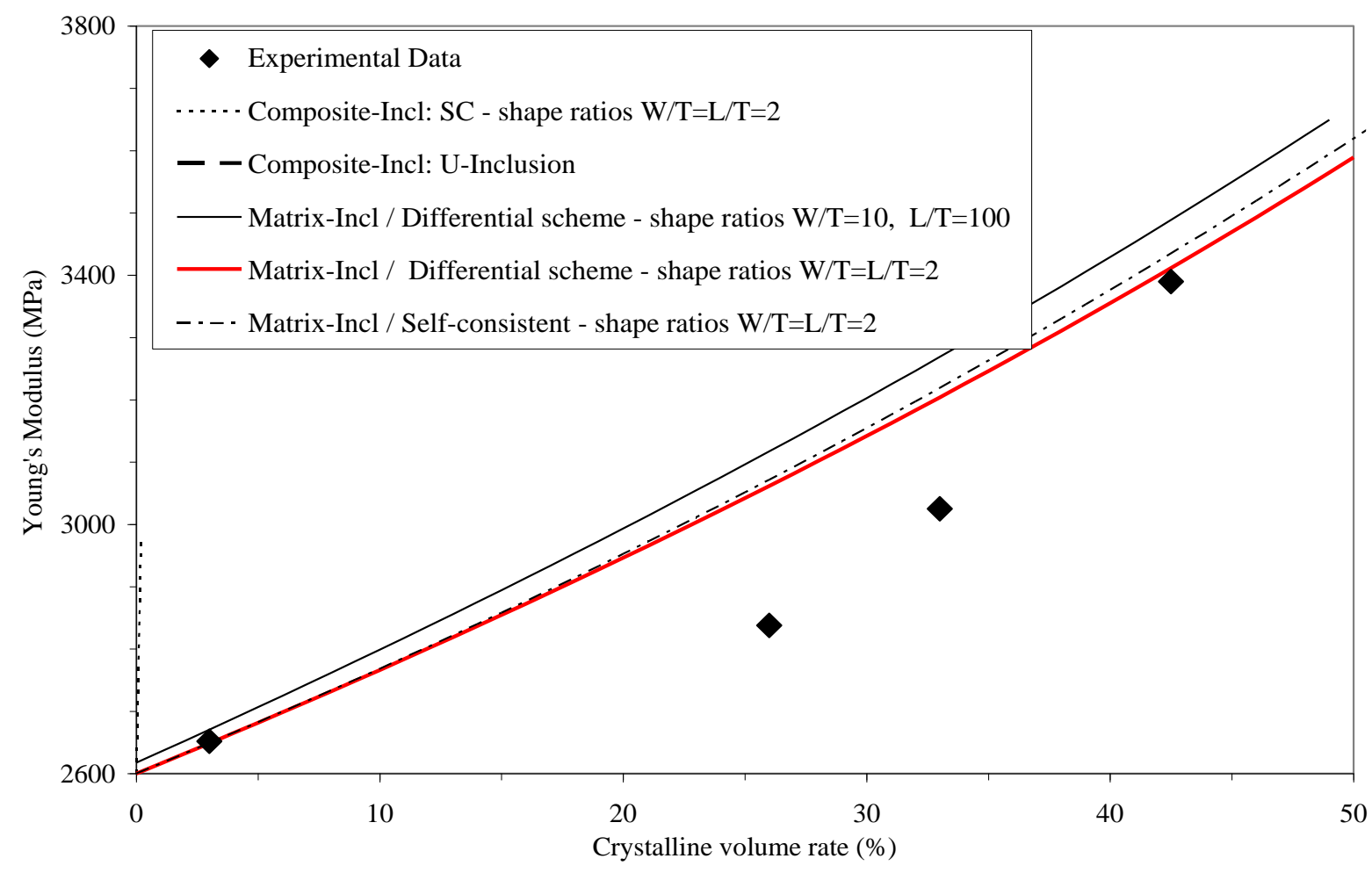

Figure 20. Predicted and experimental PET Young's modulus vs. crystallinity

As none of the models lead to really satisfactory quantitative results for the most simple case of the elastic predictions of isotropic semicrystalline materials, should not the spherulitic microstructure be better modelled? Do an aggregate of randomly oriented crystallites correctly represent this microstructure? Intuitively, it is not surprising that an aggregate of randomly oriented crystallite inclusions associated with a self-consistent scheme is stiffer than it is observed experimentally. But then a multiscale modelling should be needed.

\section{Large deformations and bottle necks}

Under large deformations, as described in the previous chapters, the initial deformation includes straining of molecular chains in the interlamellar amorphous phase which is accompanied by lamellae separation, rotation of lamellar stacks and interlamellar shear. At the yield intensive crystallographic slips are observed leading shortly to fragmentation, then to formation of fibrils and generally a disintegration of lamellae. After yielding some cavities can be are formed, and the apparition or not of disentanglements in the amorphous phase influences a lot the stress-strain behavior. All these complex mechanisms have been quite well established, but their modelling at two scales (lamella and macro-crystalline structure) is really difficult. The behavior of the crystalline behavior can be described as an elastoplastic 
[216] or elasto-viscoplastic [220] behavior to take the crystallographic slips into account. Is it sufficient to go further than yield? Today, the first bottle neck is certainly to describe the role and the behavior of the confined amorphous phase, especially if it is in the rubbery state. By modelling the dynamic linear viscoelasticity of semicrystalline PET, Diani et al. [237] showed that the mechanical properties of the confined amorphous phase is somehow not the one of the bulk. The second bottleneck is to take into account the initial crystalline macrostructure and its evolution during the large deformation. Van Dommelen et al. [183] developed a multiscale approach by combining a finite element model describing the deformation of spherulites at a mesoscopic level and a micromechanical model simulating the behavior the embedded crystalline and amorphous lamellae. The evolution of texture is described in terms of pole figures. This work is actually the most advanced to describe the behavior of semicrystalline polymers in large deformations.

\section{Phenomenological models of polymer deformation under tensile and compressive stresses.}

Plane strain compression in a channel die is kinematically very similar to drawing: the sample is extended and its cross-section decreases accordingly. However, the possibility of voids formation is limited due to the compressive component of stress. It means that the differences in true stress- true strain dependencies of drawn and plane strained polymers should be attributed to the formation and development of cavities. Slopes of the elastic region of true stress-true strain curves are similar in tension and in plane strain compression. The difference in mechanical properties of polymers sets in at yielding in tension. The scale of difference depends on a particular polymer: the yield in drawing for POM, PA 6, PP and HDPE takes place at a much lower stress than in plane strain compression. For polymers with low crystal plastic resistance: LDPEs and EOC the stresses at selected deformation ratio were very similar for both modes of sample loading. The conclusion is that the cavitation during deformation can be observed only in those polymers in which the value of negative pressure generated at yield is higher than the value of negative pressure required for cavitation. Otherwise the crystals will deform earlier relaxing the stress and cavitation will not appear.

Beyond the yield in channel die compressed POM, PA 6, PP and HDPE a short region of plastic flow is followed by a strong strain hardening while in drawing the plastic flow region is longer and strain hardening is only slightly pronounced before fracture. Nearly no 
difference in plastic flow and strain hardening is noticed for soft LDPEs and EOC in drawing and plane strain compression.

SAXS, densitometry and TEM [39] show a clear correlation of the formation of cavities in tension with a decrease of the yield stress when compared to the yield stress without cavitation (plane strain compression). It is also evident that cavitation is the reason of low strain hardening and intense chain disentanglement during drawing with cavitation. In turn, no significant disentanglement takes place during plastic deformation of polymers up to a high strain provided that there is no cavitation.

Mechanisms associated with plastic deformation of crystalline polymers can be explained better on a basis of two examples of isotactic polypropylene subjected to drawing and to plane strain compression in a channel die. The envision differences between deformation with and without cavitation are summarized in Figure 21.

The initial part of the elastic region of true stress-true strain curves is common for drawing and for plane strain compression. As the strain increases the material starts to yield in drawing at the stress level which is usually considered as the yield stress of a particular polymer and placed in product information sheets. However, the stress level at yield corresponds rather to the onset of cavitation and not necessarily to the onset of plastic deformation of crystals. With the progress of drawing the cavitational pores extend in the direction of drawing, the polymeric material between them starts to deform plastically, including now crystal plasticity mechanisms, giving rise to the long known "micronecking" phenomena and leading for large drawing ratios to a "micro-fibrillar" morphology. Along with cavitation and micronecking the chain disentanglement takes place, promoted by the formation of a large fraction of new free surfaces of voids. The chain disentanglement becomes intense, it results in loosening of the entanglement network and limits significantly the strain hardening. 


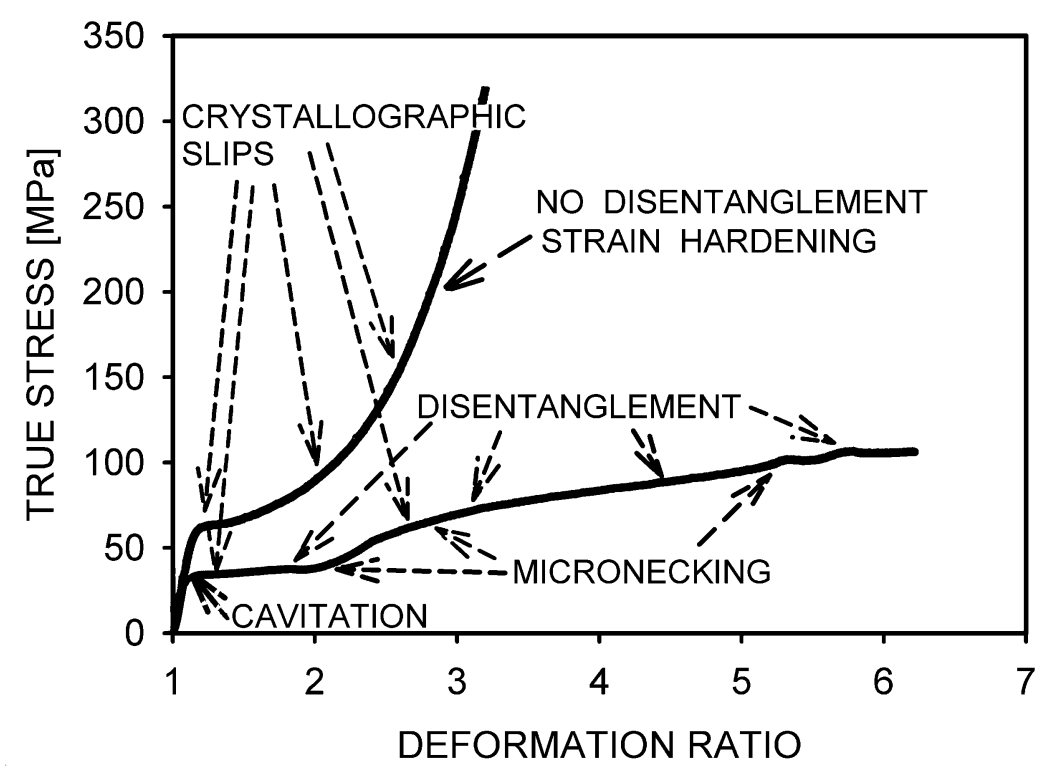

Figure 21. Mechanisms associated with plastic deformation of crystalline polymers explained on an example of isotactic polypropylene of molecular weight $3.1^{*} 10^{5}$. Redrawn after [10].

In plane strain compression the elastic region extends to a larger strain and larger stress because the formation of cavitation is not in operation while crystal plasticity needs higher resolved shear stresses to become active. When the stress reaches the level sufficient for triggering the easiest crystallographic slip ( for the example of iPP as in Figure 21 (100)[001] chain slip system at a critical resolved shear stress of $22.6 \mathrm{MPa}$ [188], also reported at $25 \mathrm{MPa}$ [185]) crystal plasticity overtakes the control of further deformation process. The real yield stress for polypropylene from Figure 21 as determined by crystal plasticity is now at the level of 50-55 $\mathrm{MPa}$.

In plane strain compression the plastic flow ends shortly and strain hardening induced by straightening of the entanglement network sets in. Since no or little chain disentanglement occurs the stress reaches very high values at a relatively low true strain.

\section{Conclusions}

The promotion of energy dissipative processes that delay or entirely suppress fracture processes originating from imperfection of internal structure or scratches and notches is enhanced by cavitation. In almost all cases cavitation either makes possible further toughening by activating other mechanisms or contributes itself to the plastic response of the polymer. The most energy dissipative processes: crazing and shear yielding occur then at a reduced stress level. 
In crystalline polymer systems the tough response, besides cavitation and crazing, is crystallographic in nature. Crystallographic slips are the main plastic deformation mechanisms that require generation and motion of crystallographic dislocations. The concepts of generation of monolithic and half-loop dislocations explain plausibly the observed yield stress dependences on crystal thickness, temperature and strain rate.

It appeared that a successful modelling of mechanical properties of crystalline polymers within elastic range requires a consideration of lamellae thickness and crystallinity but also the lamellae width and length. Varying elastic properties of the amorphous phase are to be consider when the constraints between lamellar crystals change due to differentiated solidification conditions.

\section{Literature}

1. Keller A (1968) Polymer crystals, Rep Prog Phys 31:623-704.

2. Keith H D and Padden F J Jr (1964) Spherulitic crystallization from the melt. I. Fractionation and impurity segregation and their influence on crystalline morphology, $J$ Appl Phys 35:1270-1285 .

3. Keith H D and Padden F J Jr (1964) Spherulitic crystallization from the melt. II. Influence of fractionation and impurity segregation on the kinetics of crystallization, $J$ Appl Phys 34:1286-1296.

4. Coriell S R, McFadden G B, Voorhees P W and Sekerka R F (1987) Stability of a planar interface during solidification of a multicomponent system, J Cryst Growth 82:295-302.

5. Woodruff D P (1973), The Solid-Liquid Interface, Cambridge University Press, Cambridge, U.K.

6. Calvert P D (1983) Fibrillar structures in spherulites, J Polym Sci Polym Lett Ed 21:467473.

7. Galeski A, Argon A S and Cohen R E (1987) Morphology of Nylon 6 Spherulites, Die Makromolekulare Chemie 188:1195.

8. Oleinik E F (2003) Plasticity of semicrystalline flexible-chain polymers at microscopic and mezoscopic levels, Polym Sci (Russian) Ser C 45:17-117.

9. Galeski A (2003) Strength and toughness of crystalline polymersin, Prog Polym Sci 28:1643-1699. 
10. Pawlak A and Galeski A (2005) Plastic Deformation of Crystalline Polymers: The Role of Cavitation and Crystal Plasticity, Macromolecules 38:9688-9697.

11. Hughes D J, Mahendrasingam A, Oatway W B, Heeley E L, Martin C and Fuller W (1997) A simultaneous SAXS/WAXS and stress-strain study of polyethylene deformation at high strain rates, Polymer 38:6427-6430.

12. Zhang X C, Butler M F and Cameron R E (2000) The ductile-brittle transition of irradiated isotactic polypropylene studied using simultaneous small angle X-ray scattering and tensile deformation, Polymer 41:3797-3807.

13. Liu Y and Truss R W (1994) Study of tensile yielding of isotactic polypropylene, $J$ Polym Sci B Polym Phys 32:2037-2047.

14. Kapur S, Matsushige S, Galeski A and Baer E (1978) The Effect of Pressure and Environment on the Fracture and Yield of Polymers in Advances In Research on Strength and Fracture of Materials (Ed Taplin D N R) Pergamon Press, New York pp.1079-1086.

15. Pae K D, Chiu H C, Lee J K and Kim J H (2000) Healing of stress-whitening in polyethylene and polypropylene at or below room temperature, Polym Eng Sci 40:17831795.

16. Yamaguchi M and Nitta K H (1999) Optical and acoustical investigation for plastic deformation of isotactic polypropylene/ethylene-1-hexene copolymer blends, Polym Eng Sci 39:833-840.

17. Galeski A and Bartczak Z (2003) Cavitation and cavity-free deformation of filled crystalline polymer systems, Macromol Symp 194:47-62.

18. Peterlin A and Balta-Caleja F J (1969) Plastic deformation of polypropylene. III. Smallangle x-ray scattering in the neck region, J Appl Phys 40:4238-4242.

19. Dijkstra P T S, Van Dijk D J and Huetnik J (2002) A microscopy study of the transition from yielding to crazing in polypropylene, Polym Eng Sci 42:152-160.

20. Lezak E, Bartczak Z and Galeski A (2006) Plastic deformation behavior of beta phase isotactic polypropylene in plane-strain compression at room temperature, Polymer 47:8562-8574.

21. Hiss R, Hobeika S, Lynn C and Strobl G (1999) Network stretching, slip processes, and fragmentation of crystallites during uniaxial drawing of polyethylene and related copolymers. A comparative study, Macromolecules 32:4390-4403. 
22. Butler M F, Donald A M and Ryan A J (1998) Time resolved simultaneous small- and wide-angle X-ray scattering during polyethylene deformation - II. Cold drawing of linear polyethylene, Polymer 39:39-52.

23. Wang K H, Chung I J, Jang M C, Keum J K and Song H H (2002) Deformation behavior of polyethylene/silicate nanocomposites as studied by real-time wide-angle $\mathrm{X}$ ray scattering, Macromolecules 35:5529-5539.

24. Seguela R (2007) Plasticity of semi-crystalline polymers: Crystal slip versus meltingrecrystallization, e-Polymers 032:1-20.

25. Pawlak A and Galeski A (2005) Plastic Deformation of Crystalline Polymers:The Role of Cavitation and Crystal Plasticity, Macromolecules 38:9688-9697.

26. Aboulfaraj M and G'Sell C, Ulrich B, Dahoun A (1995) Polymer 36:731-742.

27. Lee J K, Kim J H, Chu H C and Pae K D (2002) Macroscopic observation of healing process in stress-whitened polypropylene under hydrostatic pressure, Polym Eng Sci 42:2351-2360.

28. Plummer C J G, Scaramuzzino P,.Kausch H H and Phillipoz J M (2000) High temperature slow crack growth in polyoxymethylene, Polym Eng Sci 40:1306-1317.

29. Plummer C J G,Beguelin P, Grein C, Gensler R, Dupuits L, Gaillard C, Stadelmann P, Kausch H H and Manson J A E (2004) Microdeformation in heterogeneous polymers, revealed by electron microscopy, Macromol Symp 214:97-114.

30. Na B, Zhang Q, Fu Q, Men Y, Hong K and Strobl G (2006) Macromolecules 39:2584.

31. Friedrich K (1983) Crazes and shear bands in semi-crystalline thermoplastics in Advances in Polymer Sciences 52/53 (Ed Kausch H H) Springer: Berlin, pp. 225-274

32. Kausch H H, Gensler R, Grein Ch, Plummer C J G and Scaramuzzino P (1999) Crazing in semicrystalline thermoplastics, J Macromol Sci Phys B 38:803-815.

33. Jang B Z, Uhlmann D R and Van der Sande J B (1985) Crazing in polypropylene, Polym Eng Sci 25:98-104.

34. Henning S, Adhikari R, Michler G H, Balta Calleja F J and Karger-Kocsis J (2004) Micromechanical mechanisms for toughness enhancement in $\beta$-modified polypropylene, Macromo Symp 214:157-171.

35. Narisawa I and Yee A F (1993) In Materials Sciences and Technology. A Comprehensive Treatment. Vol. 12. Structure of Polymers; (Ed Thomas E L) VCH: Weinheim, pp.699-766.

36. Brown H R and Kramer E J (1980) Craze microstructure from small-angle X-ray scattering (SAXS), J Macromol Sci Phys B19:487-522. 
37. Mills P J and Kramer E J (1985) Real time small-angle X-ray scattering from polystyrene crazes during fatigue, J Mater Sci 20:4413-4420.

38. Zafeiropoulos N E, Davies R J, Schneider K, Burghammer M, Riekel Ch and Stamm M (2006) The relationship between craze structure and molecular weight in polystyrene as revealed by $\mu$ SAXS experiments, Macromol Rapid Commun 27:1689-1694.

39. Galeski A, Argon A S and Cohen R E (1988) Changes in the Morphology of Bulk Spherulitic Nylon 6 Due to Plastic Deformation, Macromolecules 21:2761-2770.

40. Argon A S (1972) In Treatise on Materials Science and Technology. Vol.1; (Ed Herman H) Academic Press: New York, pp.79.

41. Krumova M, Henning S and Michler G H (2006) Chevron morphology in deformed semicrystalline polymers, Phi Mag 86:1689-1712.

42. Thomas C, Ferreiro V, Coulon G and Seguela R (2007) In situ AFM investigation of crazing in polybutene spherulites under tensile drawing, Polymer 48:6041-6048.

43. Bucknall C B (1977) Toughened Plastics in Applied Science: London.

44. Quatravaux T, Elkoun S, G'Sell C, Cangemi L and Meimon Y (2002) Experimental characterization of the volume strain of poly(vinylidene fluoride) in the region of homogeneous plastic deformation, J Polym Sci Part B Polym Phys 40:2516-2522.

45. G'Sell C, Hiver J M and Dahoun A (2002) Experimental characterization of deformation damage in solid polymers under tension, and its interrelation with necking, Int J Solids and Structures 39:3857-3872.

46. Castagnet S, Girault S, Gacougnolle J L and Dang P (2000) Cavitation in strained polyvinylidene fluoride: Mechanical and X-ray experimental studies, Polymer 41:75237530.

47. Addiego F, Dahoun A, G'Sell Ch and Hivier J M (2006) Characterization of volume strain at large deformation under uniaxial tension in high-density polyethylene, Polymer 47:4387-4399.

48. G'Sell Ch, Bai S L and Hiver J M (2004) Polypropylene/polyamide 6/polyethyleneoctene elastomer blends. Part 2: Volume dilatation during plastic deformation under uniaxial tension, Polymer 45:5785-5792.

49. Temimi N, Burr A and Billon N (2006) In Book of Abstracts. Euromech Colloquium 487. Structure Sensitive Mechanics of Polymer Materials: Physical and Mechanical Aspects. Strasbourg 10-13.10.2006; (Eds Remond Y, Patlazhan S) Strasbourg, pp. 6768. 
50. Pawlak A (2007) Cavitation During Tensile Deformation of High Density Polyethylene, Polymer 48:1397-1409.

51. Pawlak A and Galeski A, Cavitation during Tensile Deformation of Polypropylene, Macromolecules in print DOI: 10.1021/ma0715122.

52. Bucknall C B (1978) Fracture and failure of multiphase polymers and polymer composites, Adv Polym Sci 27:121-148.

53. Kramer E J (1983) Microscopic and molecular fundamentals of crazing in Adv Polym Sci 52/53:275-334.

54. Duffo P Monasse B, Haudin J M, G'Sell C and Dahoun A (1995) Rheology of polypropylene in the solid state, J Mater Sci 30:701-711.

55. Li J X and Cheung W L (1998) On the deformation mechanisms of $\beta$-polypropylene: 1 . Effect of necking on $\beta$-phase PP crystals, Polymer 39:6935-6940.

56. Li J X, Cheung W L and Chan C M (1999) On deformation mechanisms of $\beta$ polypropylene 2. Changes of lamellar structure caused by tensile load, Polymer 40:2089-2102.

57. Li J X, Cheung W L and Chan C M (1999) On deformation mechanisms of $\beta$ polypropylene 3. Lamella structures after necking and cold drawing, Polymer 40:36413656.

58. Piorkowska E, Galeski A and Kryszewski M (1982) Heat Conduction Anisotropy of Drawn High Density Polyethylene Samples, Colloid Polym Sci 260:735-741.

59. Butler M F, Donald A M and Ryan A J (1997) Time resolved simultaneous small- and wide-angle X-ray scattering during polyethylene deformation: 1. Cold drawing of ethylene- $\alpha$-olefin copolymers, Polymer 38:5521-5538.

60. Butler M F, Donald A M and Ryan A J (1998) Time resolved simultaneous small- and wide-angle X-ray scattering during polyethylene deformation - II. Cold drawing of linear polyethylene, Polymer 39:39-52.

61. Keith H D and Padden F J Jr (1964) Spherulitic crystallization from the melt. I. Fractionation and impurity segregation and their influence on crystalline morphology, $J$ Appl Phys 35:1270.

62. Narisawa I and Ishikawa M (1990) Crazing in semicrystalline thermoplastics, $A d v$ Polym Sci 91/92:353.

63. Plummer C J G and Kausch H H (1996) Deformation and entanglement in semicrystalline polymers, J Macromol Sci Phys 35(3-4):637-657. 
64. Crist B and Peterlin A (1973) Radical formation during the tensile failure of polymer fibres, Die Makromol Chem 171:211-227.

65. Peterlin A (1975) Plastic deformation of crystalline polymers in Polymeric Materials (Ed Baer E) American Society for Metals: Metals Park, Ohio, pp, 175-195.

66. Galeski A, Bartczak, Argon A S and Cohen R E (1992) Morphological Alterations during Texture Producing Plane Strain Compression of High Density Polyethylene, Macromolecules 25: 5705-5718.

67. Pluta M, Bartczak Z and Galeski A (2000) Changes in the morphology and orientation of bulk spherulitic polypropylene due to plane strain compression, Polymer 41:22712288.

68. Bellare A, Cohen R E and Argon A S (1993) Development of texture in poly(ethylene terephthalate) by plane-strain compression, Polymer 34:1393-1403.

69. Boontongkong Y, Cohen R E, Spector M and Bellare A (1998) Orientation of plane strain-compressed ultra-high-molecular-weight polyethylene, Polymer 39:6391-6400.

70. Lin L, Argon A S (1992) Deformation resistance in oriented nylon 6, Macromolecules 25:4011-4024.

71. Lin L, Argon A S (1994) Rate mechanism of plasticity in the crystalline component of semicrystalline nylon 6, Macromolecules 27(23):6903-6914.

72. Galeski A, Argon A S and Cohen R E (1991) Morphology of Bulk Nylon 6 Subjected to Plane Strain Compression, Macromolecules 24:3953-3961.

73. Muratoglu O K, Argon A S, Cohen R E and Weinberg M (1995) Microstructural processes of fracture of rubber-modified polyamides, Polymer 36:4771-4786.

74. Muratoglu O K, Argon A S, Cohen R E and Weinberg M (1995) Microstructural fracture processes accompanying growing cracks in tough rubber-modified polyamides, Polymer 36:4787-4795.

75. Goodier J (1933) Concentration of Stress Around Spherical and Cylindrical Inclusions and Flaws, Trans ASME 55:39-42.

76. Wu S (1982) Polymer Interface and Adhesion; Marcel Dekker: New York, p. 88-92

77. Kasemura T, Yamashita N, Suzuki K, Kondo T and Hata T (1978) Studies on surface tension of polymers in relation to their structures. IV. Composition dependence of surface tension of copolymers, Kobunshi Ronbunshu 35(4):263-268.

78. Polymer Handbook, 4th. (Eds Brandrup, Immergut E H, Grulke E A) Wiley: New York, 1999. 
79. Piorkowska E and Galeski A (1993) Crystallization of Isotactic Polypropylene and High Density Polyethylene under Negative Pressure Resulting from Uncompensated Volume Change, Journal of Polymer Science Part B-Polymer Physics 31:1285-1291.

80. Pawlak A and Piorkowska E (1999) Effect of negative pressure on melting behavior of spherulites in thin films of several crystalline polymers, J Appl Polym Sci 74:1380-1385.

81. Nowacki R, Kolasinska J and Piorkowska E (2001) Cavitation during isothermal crystallization of isotactic polypropylene, J Appl Polym Sci 79:2439-2448.

82. Galeski A, Piorkowska E In Liquids Under Negative Pressure, NATO Science Series, II. Mathematics, Physics and Chemistry, vol. 84.; (Eds Imre A R, Maris H J, Williams)

83. Bowden P B, Young R J (1971) Critical Resolved Shear Stress for [001] Slip in Polyethylene, Nature 229:23-25.

84. Haudin J M (1982) Plastic deformation of semicrystalline polymers. in Plastic Deformation of Amorphous and Semi-crystalline Materials (Eds Escaig B, G'Sell C) Les Editions de Physique: Paris, 291.

85. Gaucher-Miri V and Seguela R (1997) Tensile yield of polyethylene and related copolymers: Mechanical and structural evidences of two thermally activated processes, Macromolecules 30:1158-1167.

86. Argon A S and Cohen R E (1990) Crazing and toughness of block copolymers and blends, Adv Polym Sci 91,92:301-351.

87. Kinloch A J and Young R J (1983) Fracture behaviour of polymers, Applied Sci Pub London, New York, pp. 147-178.

88. Kramer E J and Berger L L (1990) Fundamental processes of craze growth and fracture, Adv Polym Sci 91,92:1-68.

89. Olf H G and Peterlin A (1974) Cryogenic crazing of crystalline, isotactic polypropylene, J Colloid Interface Sci 47:621-635.

90. Horst J J and Spoormaker J L (1996) Mechanism of fatigue in short glass fiber reinfoced polyamide 6, Polym Eng Sci 36:2718-2726.

91. Boyer R F Feb (1975) Mechanical relaxation spectra of crystalline and amorphous polymers, Midland Macromolecular Institute Symposium on "Molecular Basis of Transitions and Relaxations, Midland, Michigan, Materials distributed by Boyer, RF during the Symposium,

92. Ferry J D (1970) Viscoelastic Properties of Polymers, New York, 2nd ed: Wiley J.

93. Galeski A (2002) Dynamic mechanical properties of crystalline polymer blends The influence of interfaces and orientation, e-Polymers 026:1-29. 
94. Orovan E (1948-49) Fracture and strength of solids, Rept Prog Phys 12:185-232.

95. Vincent P I (1960) The tough-brittle transition in Thermoplastics Polymer 1:425-444.

96. Ward I M and Hadley D W (1993) An Introduction to the mechanical properties of solid polymers, Chichester, New York: Wiley, 271-276.

97. Ward I M and Hadley D W (1993) An Introduction to the Mechanical Properties of Solid Polymers, Wiley: New York, 232-245.

98. Piorkowska E, Argon A S and Cohen R E (1990) Size effect of compliant rubbery particles on craze plasticity in polystyrene, Macromolecules 23:3838-3848.

99. Flory P J and Yoon DY (1978) Molecular morphology in semicrystalline polymers, Nature 272:226-229.

100. Gent A N and Madan S (1989) Plastic Yielding of Partially Crystalline Polymers. $J$ Polym Sc Polym Phys Ed 27:1529-1542.

101. Young R J (1976) A dislocation model for yield in polyethylene, Phil. Mag 30:86-94.

102. Seguela R (2007) Plasticity of semi-crystalline polymer: crystal slip versus meltingrecrystallization, e-Polymers, $\mathbf{0 3 2}$.

103. Lv R,.Xu W, Na B, Zhang Q and Fu Q (2008) Large Tensile Deformation Behavior of Oriented High-Density Polyethylene: A Correlation between Cavitation and Lamellar Fragmentation, J Polym Sci, Polym Phys 46:1202-1206).

104. Peterlin A (1971) Radical formation and fracture of highly drawn crystalline polymers, J Macromol. Sci Phys 6:490-508.

105. Kelly A and Groves G W (1970) Crystallography of Crystal Defects, Longman: London, UK, 1-275

106. Cottrell A H (1953) Dislocation and Plastic Flow in Crystals, Oxford Uni Press: London,; Chapter 3.

107. Bartczak Z, Argon A S and Cohen R E (1992) Deformation Mechanisms and Plastic Resistance in Single-Crystal-Textured High-Density Polyethylene, Macromolecules 25:5036-5053.

108. Bowden P B and Young R J (1974) Deformation mechanisms in crystalline polymers, $J$ Mater Sci 9:2034-2051.

109. Shinozaki D and Groves G (1973) The plastic deformation of oriented polypropylene: tensile and compressive yield criteria, J. Mater Sci 8:71-78.

110. Caddell R M and Raghava R S, Atkins A G (1973) A yield criterion of anisotropic and pressure dependent solids such as oriented polymers, J Mater Sci 8:1641-1646. 
111. Aboulfaraj M and G'Sell C, Ulrich B,Dahoun A (1995) In situ observation of the plastic deformation of polypropylene spherulites under uniaxial tension and simple shear in the scanning electron microscope, Polymer 36:731-742.

112. Bartczak $Z$ and Martuscelli E (1997) Orientation and properties of sequentially drawn films of an isotactic polypropylene; hydrogenated oligocyclopentadiene blends, Polymer 38:4139-4149.

113. Bartczak Z and Galeski A (1999) Yield and Plastic Resistance of a -Crystals of Isotactic Polypropylene, Polymer 40:3677-3684.

114. Lee B J, Argon A S, Parks D M, Ahzi S and Bartczak Z (1993) Simulation of Large Strain Plastic Deformation and Texture Evolution in High-Density Polyethylene,. Polymer 34:3555-3575.

115. Lin L and Argon A S (1994) Review: Structure and plastic deformation of polyethylene, J Mater Sci 29:294-323.

116. Bellare A, Argon A S and Cohen R E (1993) Development of texture in poly(ethylene terephthalate) by plane-strain compression, Polymer 34:1393-1403.

117. Eling B, Gogolewski S and Pennings A J (1982) Biodegradable materials of poly(LLactic acid): 1 Melt-spun and solution n-spun fibres, Polymer 23:1587-1593.

118. Chen X, Galeski A and Michler G H (2006) Morphological alteration of polyamide 6 subjected to high plane-strain compression, Polymer 4:, 3171-3185.

119. Lezak E, Bartczak Z and Galeski A (2006) Plastic deformation behavior of $\beta$ phase isotactic polypropylene in plane-strain compression at room temperature, Polymer, 47:8562-8574.

120. Lezak E, Bartczak Z and Galeski A (2006) Plastic deformation of the $\gamma$ phase isotactic polypropylene in plane-strain compression, Macromolecules 39(14):4811-4819.

121. Lezak E, Bartczak Z (2007) Plastic Deformation of the $\gamma$ Phase Isotactic Polypropylene in Plane-Strain Compression at Elevated Temperatures, Macromolecules 40(14):49334941.

122. Lewis D, Wheeler E J, Maddams W. F and Preedy J E (1972) Comparison of twinning produced by rolling and annealing in high- and low-density polyethylene, J Polym Sci A-2 Notes 10:369-373.

123. Bartczak Z (2002) Deformation of high density polyethylene produced by rolling with side constraints. I Orientation behavior, J Appl Polym Sci 86:1396-1404. 
124. Young R J and Bowden P B (1974) Twinning and martensitic transformations in oriented high-density polyethylene, Phil Mag 29:1061-1073.

125. Kryszewski M, Pakula T, Galeski A, Milczarek P and Pluta M. (1978) Über einige Ergebnisse der Untersuchung der Korrelation zwischen der Morphologie und den mechanischen Eigenschaften von kristallinen Polymeren, Faserforschung und Textiltech 29:76-85.

126. Mirabella F M Jr (1987) Determination of the crystalline and noncrystalline molecular orientation in oriented polypropylene by infrared spectroscopy, J Polym Sci Part B: Polym Phys 25: 591-602.

127. Burton W K, Cabrera N and Frank F C (1949) Role of Dislocations in Crystal Growth Nature 163:398.

128. Frank F C (1949) Influence of dislocations on crystal growth, Farad Soc Discuss, Crystal Growth 5:48.

129. Geil P H (1963) Polymer single crystals, New York: Interscience

130. Wunderlich B (1973) Macromolecular Physics, Crystal Structure,. Morphology, Defects, Chapter 4.1.4, Academic Press, New York.

131. Keith H D and Chen W Y (2002) On the origins of giant screw dislocations in polymer lamellae, Polymer 43:6263-6272.

132. Schultz J M and Kinloch D R (1969) Transverse screw dislocations: A source of twist in crystalline polymer ribbons, Polymer 10:271-278.

133. Duan Y, Zhang Y, Yan S and Schultz JM (2005) In situ AFM study of the growth of banded hedritic structures in thin films of isotactic polystyrene, Polymer 46:9015-9021.

134. Toda A, Arita T and Hikosaka M (2001) Three-dimensional morphology of PVDF single crystals forming banded spherulites, Polymer 42:2223-2233.

135. Ikehara T, Jinnai H, Kaneko T, Nishioka H and Nishi T, (2007) Local Lamellar Structures in Banded Spherulites Analyzed by Three-Dimensional Electron Tomography, J Polym Sci, Polym Phys 45:1122-1125 .

136. Toda A, Okamura M, Hikosaka M and Nakagawa Y (2005) Three-dimensional shape of polyethylene single crystals grown from dilute solutions and from the melt, Polymer 46:8708-8716.

137. Wilhelm H, Paris A, Schafler E, Bernstorff S, Bonarski J, Ungar T and Zehetbauer M.J (2004) Evidence of dislocations in melt-crystallized and plastically deformed polypropylene, Materials Science and Engineering A 387-389:1018-1022. 
138. Schafler E, Zehetbauer M and UngarT (2001) Measurement of screw and edge dislocation density by means of X-ray Bragg profile analysis, Materials Science and Engineering A319-321:220-223.

139. Keith H D and Passaglia E (1964) Dislocations in polymer crystals, J Res Natl Bur Stand (US);68A:513-518.

140. Peterson J M (1966) Thermal Initiation of Screw Dislocations in Polymer Crystal Platelets, J Appl Phys 37:4047-4050 .

141. Peterson J M (1968) Peierls Stress for Screw Dislocations in Polyethylene, J Appl Phys 39:4920-4928.

142. Shadrake L G and Guiu F (1976) Dislocations in polyethylene crystals: line energies and deformation modes, Philos Mag 34:565.

143. Young R J (1988) Screw dislocation model for yield in polyethylene, Mater Forum 11:210-216.

144. Lin L and Argon A S (1994) Rate Mechanism of Plasticity in the Crystalline Component of Semicrystalline Nylon 6, Macromolecules 27(23):6903-6914.

145. Galeski A, Bartczak Z, Argon A S and Cohen R.E (1992) Morphological alterations during texture-producing plastic plane strain compression of high-density polyethylene, Macromolecules 25(21):5705-5718.

146. Kazmierczak T, Galeski A and Argon A S (2005) Plastic deformation of polyethylene crystals as a function of crystal thickness and compression rate, Polymer 46(21): 89268936.

147. Young R J (1974) Dislocation model for yield in polyethylene, Philos Mag 30(1):85-94

148. Crist B, Fisher C J and Howard P (1989) Mechanical properties of model polyethylenes: tensile elastic modulus and yield stress, Macromolecules 22(4):1709-1718.

149. Darras O and Séguéla R (1993) Tensile yield of polyethylene in relation to crystal thickness, Journal of Polymer Science Part B: Polymer Physics 31(7):759-766.

150. O'Kane W J, Young R J and Ryan A J (1995) The role of dislocations in the yield of polypropylene, J Macromol Sci Phys 34:427-458.

151. Shadrake L G and Gui F (1976) Dislocations in polyethylene crystals: line energies and deformation modes, Philos Mag 34: 565-581.

152. Shadrake L G and Gui F 1979, Elastic line energies and line tensions of dislocations in polyethylene crystals, Philos Mag 39:785-796

153. Bacon D J and Tharmalingam K (1983) Computer simulation of polyethylene crystals. Part 3 The core structure of dislocations, J Mater Sci 18:884-893 
154. Argon A S, Galeski A and Kazmierczak T (2005) Rate mechanisms of plasticity in semi-crystalline polyethylene, Polymer 46:11798-11805.

155. Bartczak Z, Cohen R E and Argon A S (1992) Evolution of Crystalline Texture of High-Density Polyethylene During Uniaxial Compression, Macromolecules 25:46924704.

156. Peierls R (1940) The size of dislocations, Proc Phys Soc 289(52):34-37.

157. Nabarro F R N (1947) Dislocations in a simple cubic lattice, Proc Phys Soc 59:256-272.

158. Honeycomb R W K (1968) The Plastic Deformation of Metals; Edward Arnold Ltd: London, UK 41-47.

159. Frank F C and Read W T (1950) Multiplication processes for slow moving dislocations. Phys Rev 79:722-723.

160. Shadrake L G and Guiu F (1974) Dislocation in polyethylene crystals: Line energies and deformation modes, Phil Mag 34:565-581.

161. Crist B (1989) Yielding of semicrystalline polyethylene: a quantitative dislocation model, Polym Comm 30:69-71.

162. Darras O and Seguela R (1993) Tensile Yield of Polyethylene in Relation to Crystal Thickness. J Polym Sci Part B Polym Phys 31:759-766.

163. Brooks N W J, Ducket R A and Ward I M (1998) Temperature and Strain-Rate Dependence of Yield Stress of Polyethylene, J Polym Sci Part B Polym Phys 36:21772189.

164. Seguela R (2002) Dislocation Approach to the Plastic Deformation of Semicrystalline Polymers: Kinetic Aspects for Polyethylene and Polypropylene, J Polym Sci Part B Polym Phys 40:593-601.

165. Kazmierczak T and Galeski A (2002) Transformation of Polyethylene Crystals by High Pressure, J Appl Polym Sci 86:1337-1350.

166. Kazmierczak T and Galeski A (2003) Plastic deformation of polyethylene crystals as a function of crystal thickness and compression rate. Proceedings of 12th International Conference on Deformation, Yield and Fracture of Polymers, Cambridge: UK, Apr7-10.

167. Brooks N W J and Mukhtar M (2000) Temperature and stem length dependence on the yield stress of polyethylene, Polymer 41:1475-1480.

168. Petermann J and Gleiter H (1972) Direct observation of dislocations in polyethylene crystals, Philos Mag 25:813-816.

169. Peterson J M (1966) Thermal Initiation of Screw Dislocations in Polymer Crystal Platelets, J Appl Phys 37:4047-4050 . 
170. Xu G, Argon A S and Ortiz M (1995) Nucleation of dislocations from crack tips under mixed modes of loading: Implications for brittle against ductile behaviour of crystals, Philos Mag A72:415-451.

171. Xu G, Argon AS and (1997) Ortiz M. Critical configurations for dislocation nucleation from crack tips, Philos Mag A75:341-367.

172. Xu G and Zhang C (2003) Analysis of dislocation nucleation from a crystal surface based on the Peierls-Nabarro dislocation model, J Mech Phys Solids 51:1371-1394.

173. Xu G (2002) Energetics of nucleation of half dislocation loops at a surface crack, Philos Mag A;82:3177-3185.

174. Lee B J, Argon A S, Parks D M, Ahzi S and Bartczak Z (1993) Simulation of large strain plastic deformation and texture evolution in high density polyethylene, Polymer 34:3555-3575.

175. van Dommelen J A W, Parks D M, Boyce M C, Brekelmans W A M and Baaijens F P T (2003) Micromechanical modeling of intraspherulitic deformation of semicrystalline polymers, Polymer 44:6089-6101.

176. van Dommelen J A W, Parks D M, Boyce M C, Brekelmans W A M, Baaijens F P T (2003) Micromechanical modeling of the elasto-viscoplastic behavior of semicrystalline polymers, J Mech Phys Solids 51:519-541.

177. Shinozaki D and Groves G W (1973) The plastic deformation of oriented polypropylene: tensile and compressive yield criteria, J Mater Sci 8:71-78.

178. Kaito A, Nakayama K and Kanetsuna H (1986) Roller drawing of polyoxymethylene, $J$ Appl Polym Sci 32:3499-3513.

179. Kazmierczak T and Galeski A (2003) In Proceedings of VIth ESAFORM Conference on Material Forming; Univ. Salerno, Italy.

180. Bartczak Z and Galeski A (1997) Plastic resistance of isotactic polypropylene $\square$-crystals in bi-oriented polypropylene/hydrogenated oligo(cyclopentadiene)blends, Fibres and Textiles East Eur 5:32-37.

181. Basset DC (1981) Principles of Polymer Morphology, Cambridge University Press, Cambridge, UK.

182. Ward IM (1997) Structure and Properties of Oriented Polymers, Chapman\&Hall, London, UK

183. Fujiyama M and Wakino T (1991) Structures and properties of injection moldings of crystallization nucleator-added polypropylenes. I. Structure-property relationships. $J$ Appl Polym Sci 42: 2739-2747. 
184. Pople JA, Mitchel GR, Sutton SJ,Vaughan AS and Chai CK (1999) The development of organized structures in polyethylene crystallized from a sheared melt, analyzed by WAXS and TEM, Polymer 40: 2769-2777.

185. Mendoza R, Régnier G, Seiler W and Lebrun JL (2003) Spatial distribution of molecular orientation in injected molded iPP : influence of processing conditions, Polymer 44: 3363-3373.

186. Koscher E and Fulchiron R (2002) Influence of shear on polypropylene crystallization: morphology, development and kinetics. Polymer 43: 6931-6942.

187. Phillips R, Herbert G, News J and Wolkowicz M, (1994) High modulus polypropylene: Effect of polymer and processing variables on morphology and properties, Polym Eng Sci 34: 1731-1743.

188. Christensen RM (1991) Mechanics of Composite Materials, Krieger Publishing Company, Malabar, Florida.

189. Nemat-Nasser S, Hori M. Micromechanics (1999) Overall Properties of Heterogeneous Materials, Second revision edition, Elsevier Science BV, Amsterdam.

190. Milton GN (2002) Theory of Composites, Cambridge University Press, Cambridge, UK.

191. Steenkamer DA and Sullivan JL (1999) The performance of calcium carbonate filled, random fiber composites, Polym Comp 20: 392-405.

192. Tucker III CL and Liang E (1999) Stiffness predictions for unidirectional short-fiber composites: Review and evaluation, Comp Sci Techn 59: 655-671.

193. Zeng QH, Yu AB and Lu GQ (2008) Multiscale modeling and simulation of polymer nanocomosites, Prog Polym Sci 33: 191-269.

194. Ladeveze P and Lubineau G (2002) An enhanced mesomodel for laminates based on micromechanics, Comp Sci Techn 62: 533-541.

195. Liu Y, Gilormini P and Castaneda PP (2003) Variational self-consistent estimates for texture evolution in viscoplastic polycrystals, Acta Mater 51: 5425-5437.

196. Hill R. (1963) Elastic properties of reinforced solids: Some theoretical principles, $J$ Mech Phys Solids 11: 357-372.

197. Mori T, Tanaka K. (1973) Average stress in matrix and average elastic energy of materials with misfitting inclusions, Acta Metall 21:571-574.

198. Eshelby JD (1957) The determination of the elastic field of an ellipsoidal inclusion and related problems, Proc Royal Soc Lond A241: 376-396. 
199. Gilormini P and Brechet Y (1999) Syntheses: Mechanical properties of heterogeneous media: Which material for which model? Which model for which material? Mod Sim Mat Sci Eng 7: 805-816.

200. Viana JC, Cunha AM and Billon N (2001) The effect of skin thickness and spherulite size on the mechanical properties of injection moldings, J Mater Sci 36: 4411-4418.

201. Sawyer LC, Chen RT, Jamieson MG, Musselman IH and Russel PE (1993) The fibrillar hierarchy in liquid crystalline polymers, J Mater Sci 28: 225-238.

202. Takayanagi M, Imada K and Kajiyama T (1967) Mechanical properties and fine structure of drawn polymers, J Polym Sci Part C: Polym Symp 159: 263-281.

211. Halpin JC, Kardos JL.(1972) Moduli of Crystalline Polymers Employing Composite Theory, J Appl Phys 43: 2235-2241.

212. Tsai SW, Pagano NJ (1968) Invariant Properties of Composite Materials, Proceedings of Composite Materials Workshop, Technomic Publishing (Eds Tsai SW, Halpin JC, Pagano NJ), Stamford, Connecticut, pp. 233-253.

213. Phillips PJ, Patel J (1978)The influence of morphology on the tensile properties of polyethylenes, Polym Eng Sci 18: 943-950.

214. Janzen J (1992) Elastic moduli of semicrystalline PE compared with theoretical micromechanical models for composites, Polym Eng Sci 32: 1242-1254.

215. Parks DM, Ahzi S (1990) Polycrystalline plastic deformation and texture evolution for crystals lacking five independent slip systems, J Mech Phys Solids 38: 701-724.

216. Dahoun A, Aboulfaraj M, G'Sell C, Molinari A, Canova GR (1995) Plastic behavior and deformation textures of poly(etherether ketone) under uniaxial tension and simple shear, Polym Eng Sci 35: 317-330.

217. G’Sell C, Dahoun A, Favier V, Hiver JM, Philippe MJ, Canova GR (1997) Microstructure transformation and stress-strain behavior of isotactic polypropylene under large plastic deformation, Polym Eng Sci 37: 1702-1711.

218. Lee BJ, Parks DM, Ahzi S (1993) Micromechanical modeling of large plastic deformation and texture evolution in semi-crystalline polymers, J Mech Phys Solids 41: 1651-1687.

219. Lee BJ, Argon AS, Parks DM, Ahzi S (1993) Simulation of large strain plastic deformation and texture evolution in high density polyethylene, Polymer 34: 35553575 
220. Nikolov S, Doghri I, Pierard O, Zealouk L, Goldberg A (2002) Multi-scale constitutive modeling of the small deformations of semi-crystalline polymers, J Mech Phys Solids 50: $2275-2302$.

221. Bedoui F, Diani J, Regnier G, Seiler W (2004) Micromechanical modeling of elastic properties in polyolefins, Polymer 45, 2433-2442.

222. Bedoui F, Diani J, Regnier G (2004) Micromechanical modeling of isotropic elastic behavior of semicrystalline polymers, Acta Mater 54: 1513-1523.

223. Tashiro K, Kobayashi M (1996) Molecular theoretical study of the intimate relationships between structure and mechanical properties of polymer crystals, Polymer 37: 17751786.

224. Tashiro K, Kobayashi M, Tadokoro H. (1992) Vibrational Spectra and Theoretical Three-Dimensional Elastic Constants of Isotactic Polypropylene Crystal: An Important Role of Anharmonic Vibrations, Polymer J 24: 899-916.

225. Choy CL, Leung WP (1985) Elastic moduli of ultradrawn polyethylene, J Polym Sci Polym Phys Ed 23: 1759-1780.

226. Hine PJ, Ward IM (1996) Measuring the elastic properties of high-modulus fibres, $J$ Mater Sci 31: 371-379.

227. Rutledge GC (1997) Thermomechanical properties of the crystal phase of poly(ethylene terephthalate) by molecular modeling, Macromolecules 30: 2785-2791.

228. Matsuo M, Sawatari C (1990) Morphological and Mechanical Properties of Poly(ethylene terephthalate) Gel and Melt Films in Terms of the Crystal Lattice Modulus, Molecular Orientation, and Small Angle X-Ray Scattering Intensity Distribution, Polym J 22:518-538.

229. Gorbunov AA, Skvortsov AM (1995), Statistical properties of confined molecules, Adv Coll Interf Sci 62:31-108.

230. Guan X, Pitchumani R (2004) A micromechanical model for the elastic properties of semicrystalline thermoplastic polymers, Polym Eng Sci 44: 433 -451.

231. Ahzi S, Parks DM, Argon AS (1995) Estimates of the overall elastic properties in semicrystalline plymers. In current research in the thermo-mechanics of polymers in the rubbery-glassy range, ASME, Ed. Negahban M, AMD 203: 31-40.

232. Gray RW, McCrum NG (1969) Origin of the $\gamma$ relaxations in polyethylene and polytetrafluoroethylene, J Polym Sci Part A-2 Polym Phys 7: 1329-1355.

233. Boyd RH (1983) The mechanical moduli of lamellar semicrystalline polymers, J Polym Sci. Polym Phys Ed 21: 493-504. 
234. Spitalsky Z, Bleha T (2003) Elastic moduli of highly stretched tie molecules in solid polyethylene, Polymer 44: 1603-1611.

235. Flory PJ. Principles of Polymer Chemistry, Cornell University Press, Ithaca NY, 1953.

236. Wu S (1989) Chain structure and entanglement, J Polym Sci Polym Phys Ed 27: 723741.

237. Diani J, Bedoui F, Regnier G (2008) On the relevance of the micromechanics approach for predicting the linear viscoelastic behavior of semicrystalline PET, Mat Sci. Eng A 475: 229-234. 